\title{
Persistence in seasonally varying predator-prey systems via the basic reproduction number ${ }^{1}$
}

\author{
Maurizio Garrione ${ }^{\mathrm{a}}$, Carlota Rebelo ${ }^{\mathrm{b}, *}$ \\ ${ }^{a}$ Dipartimento di Matematica ed Applicazioni, Università di Milano-Bicocca, Via Cozzi 55, 20125 Milano, Italy \\ ${ }^{b}$ Centro de Matemática, Aplicações Fundamentais e Investigação Operacional, Faculdade de Ciências, Universidade \\ de Lisboa, Campo Grande, Edificio C6, piso 2, 1749-016, Lisboa, Portugal
}

\begin{abstract}
We study persistence in general seasonally varying predator-prey models. Using the notion of basic reproduction number $R_{0}$ and the theoretical results proved in [23] in the framework of epidemiological models, we show that uniform persistence is obtained as long as $R_{0}>1$. In this way, we extend previous results obtained in the autonomous case for models including competition among predators, prey-mesopredator-superpredator models and Leslie-Gower systems.
\end{abstract}

Keywords: uniform persistence, basic reproduction number, predator-prey systems.

2010 MSC classification: 92D25.

\section{Introduction}

The importance of seasonality in biological systems is unquestionable [20, 22, 25, 28, with almost no exceptions, the dynamics of population communities takes place in periodically varying environments. The main cyclic factors acting in this perspective are both of natural type and human-induced.

Of course, a periodically varying environment can be encountered in several models of different nature, like ecological (e.g., predator-prey) systems and epidemiological ones. Seasonality can be responsible for a wide variety of different complex dynamical features, such as multi-year cycles and subharmonics, quasi-periodic solutions and chaotic behaviors. In particular, it is very natural to wonder, as a first question, if a system exhibiting $T$-periodicity in the coefficients $(T>0)$ will display periodic dynamics, for instance searching for $T$-periodic solutions.

Another basic question about biological models involves the long time survival of the species: in particular, we may wonder whether the considered system is uniformly persistent with respect to one (or more) species, namely the number of individuals in that compartment (resp. those compartments) stays positive and bounded away from zero for any positive time. This issue is particularly common

\footnotetext{
${ }^{1}$ Electronic version of an article published as Persistence in seasonally varying predator-prey systems via the basic reproduction number in Nonlinear Analysis: Real World Applications, Volume 30 (2016), pp. 7398, DOI 10.1016/j.nonrwa.2015.11.007, https://www.sciencedirect.com/science/article/abs/pii/S1468121815001510 (homepage https://www.journals.elsevier.com/nonlinear-analysis-real-world-applications/).

* Corresponding author

Email addresses: maurizio.garrione@unimib.it (Maurizio Garrione), mcgoncalves@fc.ul.pt (Carlota Rebelo)

${ }^{1}$ M. Garrione wishes to acknowledge the support of the Project PTDC/MAT/113383/2009 during his stay in Lisbon and the support of GNAMPA - Gruppo Nazionale per l'Analisi Matematica, la Probabilità e le loro Applicazioni, Italy.

${ }^{2}$ Supported by Fundação para a Ciência e Tecnologia, PEst, OE/MAT/UI0209/2011 project PTDC/MAT/113383/2009 and UID/MAT/04561/2013.
} 
in epidemiology, where one usually wants to predict if a disease will go towards extinction or if it will remain endemic. Many authors dealt with this problem giving conditions which guarantee uniform persistence in seasonally forced epidemiological models, see for example [23, 24, 32, and the references therein.

In this paper, we will focus on the study of persistence for predator-prey models subject to seasonality (see, e.g., [9, 20, 25]). As a first remark, notice that it is very frequent - and it will also be one of our starting points - that the dynamics in the prey space exhibits a stable periodic orbit in absence of the predator (for instance, this is a classical fact if the function describing the internal growth of the preys is a logistic one). It is therefore natural to study the linearized system around this orbit, trying to understand the effect of the introduction of a predator. We will employ the usual approach and techniques coming from epidemiology 2, 23, in particular we will recall the notion of basic reproduction number $R_{0}$ and prove that, under suitable conditions, the predators will go extinct if $R_{0}<1$ and will persist if $R_{0}>1$. As far as we know, this kind of approach is not common in ecology, exceptions are, for example, the papers [12, 13, dealing however with autonomous systems. In autonomous epidemiological models, the basic reproduction number is defined as the expected number of new infectious produced by a single infectious individual, when introduced into a completely susceptible population. For those systems, many classical results state that the threshold value of $R_{0}$ in order to determine the qualitative dynamical features of the disease spread is 1: if $R_{0}<1$, then the disease will eventually disappear, while if $R_{0}>1$, it will persist inside the population. In these cases, $R_{0}$ can usually be easily computed starting from the coefficients appearing in the equations. On the contrary, it was only in the last years that the concept of basic reproduction number was successfully extended to nonautonomous models 2, 32, being defined as the spectral radius of a suitable operator associated with the model. This approach was proved to be quite effective, allowing to obtain several persistence results for nonautonomous systems coming from epidemiology 23, 24, 32].

Throughout the present paper, roughly speaking, $R_{0}$ may be thought about as the number of predators one predator gives rise during its life, when introduced in a prey population. For periodic predator-prey models, this will be done adapting the definitions given in [2, 32. However, we will be able to proceed a little step further; indeed, not necessarily the population with respect to which persistence is proved corresponds to the predator one, in fact its choice may be not uniquely determined. Nevertheless, thanks to the generality of the setting in 2, 32, we will be allowed to disregard whether a compartment is made up by preys or predators, basing our choice only on the dynamics of the system in absence of a certain number of compartments. Indeed, there are examples in which there exists a stable periodic orbit in the space of predators when preys do not exist, and not vice versa (see Section 5). Hence, in this case, preys and predators change role in the definition of $R_{0}$. This makes the approach sufficiently flexible and suitable to be applied to a quite large class of models, allowing to find new results for the nonautonomous case, as well as different proofs (often less laborious) of the results already present for autonomous models; in any case, we will always show that $R_{0}>1$ implies uniform persistence, and $R_{0}<1$ leads to extinction. Incidentally, notice that, when $R_{0}>1$, the existence of a periodic motion will follow as a consequence of some results in [27.

The abstract result which will be exploited in our statements, formulated for a general first order system, is given in Section 2 2 in Sections 3 and 4, we will apply it to infer results about general models of predator-prey type, in presence of one or more predators, respectively. Finally, in Section 5 we will consider a Leslie-Gower model where the role of preys and predators in the definition of $R_{0}$ is interchanged. We will constantly explore, after the statements, corresponding models which 
can be dealt with using our method.

\section{Some abstract results}

We begin this section with two results corresponding to [23, Theorems 2 and 4], stated in the framework of general systems in $\mathbb{R}^{n}$ including predator-prey ones. We give such statements to make a bridge between the epidemiological and the general population dynamics settings. In Theorem 2.3 and Proposition 2.4. we will explicitly see the central role played by the basic reproduction number $R_{0}$ and the effectiveness of our approach.

We consider $n(n \geq 2)$ populations which interact one with the other, some being preys and some predators. We denote by $x=\left(x_{1}, \ldots, x_{n}\right)^{T} \geq 0$ the vector whose $i$-th component corresponds to the $i$-th population. We will divide the compartments into two types:

- the first $m$ populations, $x_{1}, \ldots, x_{m}, 1 \leq m \leq n-1$ which, under some circumstances, will invade the other $n-m$ populations;

- the last $n-m$ populations, which, in the absence of the other $m$, have the following behaviour: there exists a periodic orbit

$$
x^{*}(t)=\left(0, \ldots, 0, x_{m+1}^{*}(t), \ldots, x_{n}^{*}(t)\right)
$$

which attracts all the nontrivial solutions in the space where the first $m$ species are absent. More precisely, setting

$$
\mathbb{R}_{+}^{n}=\left\{x \in \mathbb{R}^{n}: x_{i} \geq 0, i=1, \ldots, n\right\},
$$

we can define

$$
X_{s}:=\left\{x \in \mathbb{R}_{+}^{n}: x_{i}=0, i=1, \ldots, m\right\}
$$

and assume that $x^{*}(\cdot)$ is globally asymptotically stable in $X_{s} \backslash\{O\}$, where $O=(0, \ldots, 0)$.

Often, $\left\{x_{1}, \ldots, x_{m}\right\}$ will be predators and $\left\{x_{m+1}, \ldots, x_{n}\right\}$ will be preys.

We now introduce some notation in order to formulate the equations governing our model.

- For $1 \leq i \leq m$, let $\mathcal{F}_{i}(t, x)$ denote the input rate in the $i$-th compartment of individuals which do not immigrate from a compartment $x_{j}, j=1, \ldots, m, j \neq i$. Such an input rate corresponds either to the product of the interaction between $x_{i}$ and another population $x_{k}$ (with $k=1, \ldots, n$ ), or to new births; in any case, it consists in something which is created, not a simple flow of the same individuals between different populations.

- For $1 \leq i \leq n, \mathcal{V}_{i}^{+}(t, x)$ will denote the input rate in the $i$-th compartment of individuals by all other means. More precisely, for $1 \leq i \leq m, \mathcal{V}_{i}^{+}(t, x)$ are transfers of individuals within the first $m$ compartments, while for $m+1 \leq i \leq n, \mathcal{V}_{i}^{+}(t, x)$ are either births or transfers from other compartments.

- Finally, for $1 \leq i \leq n$, let $\mathcal{V}_{i}^{-}(t, x)$ denote the rate of transfer out of compartment $i$, such as deaths or transfers to any $j$-th compartment, $j \neq i$.

In this setting, the dynamical population model is governed by the nonautonomous ordinary differential system:

$$
x_{i}^{\prime}(t)=\mathcal{F}_{i}(t, x)-\mathcal{V}_{i}(t, x)=: f_{i}(t, x), \quad i=1, \ldots, n,
$$


where we set

$$
\mathcal{F}_{i}(t, x)=0 \quad \text { for } m+1 \leq i \leq n,
$$

and $\mathcal{V}_{i}=\mathcal{V}_{i}^{-}-\mathcal{V}_{i}^{+}$. As in [23, 32], we assume throughout the paper the following conditions:

$\left(A_{1}\right)$ For each $1 \leq i \leq n$, the functions $\mathcal{F}_{i}, \mathcal{V}_{i}^{+}, \mathcal{V}_{i}^{-}$are nonnegative and continuous on $\mathbb{R} \times \mathbb{R}_{+}^{n}$ and continuously differentiable with respect to $x$.

$\left(A_{2}\right)$ There is a positive real number $T$ such that, for each $1 \leq i \leq n$, the functions $\mathcal{F}_{i}, \mathcal{V}_{i}^{+}, \mathcal{V}_{i}^{-}$are $T$-periodic in $t$-variable.

$\left(A_{3}\right)$ If $x_{i}=0$ then $\mathcal{V}_{i}^{-}=0$.

$\left(A_{4}\right)$ If $x \in X_{s}$, then $\mathcal{F}_{i}=\mathcal{V}_{i}^{+}=0$ for $1 \leq i \leq m$.

Such a setting was originally introduced in the paper [30] for autonomous compartmental models of disease transmission. The structural assumptions $\left(A_{1}\right)$ and $\left(A_{2}\right)$ simply say that each function denotes a nonnegative transfer of individuals and that the environment modeled is a periodic one, while $\left(A_{3}\right)$ says that there cannot be transfers out of empty compartments. If we think to $x_{1}, \ldots, x_{m}$ as the predators and $x_{m+1}, \ldots, x_{n}$ as the preys, as it will be usual, $\left(A_{4}\right)$ means that if the system is predator-free at the beginning, it remains predator-free. Further, we will assume the following:

$\left(A_{5}\right)$ System (1) has a unique $T$-periodic solution in $X_{s}$, denoted by

$$
x^{*}(t)=\left(0, \ldots, 0, x_{m+1}^{*}(t), \ldots, x_{n}^{*}(t)\right)^{T},
$$

with $x_{i}^{*}(t)>0 \forall t \in[0, T]$ for at least one index $i \in\{m+1, \ldots, n\}$.

Notice now that, as in [23, 32], we have

$$
D_{x} \mathcal{F}\left(t, x^{*}(t)\right)=\left[\begin{array}{cc}
F(t) & 0 \\
0 & 0
\end{array}\right], \quad D_{x} \mathcal{V}\left(t, x^{*}(t)\right)=\left[\begin{array}{cc}
V(t) & 0 \\
J(t) & -M(t)
\end{array}\right],
$$

where $\mathcal{F}=\left(\mathcal{F}_{i}(t, x)\right)_{i=1, \ldots, n}^{T}$ and $\mathcal{V}=\left(\mathcal{V}_{i}(t, x)\right)_{i=1, \ldots, n}^{T}$ are two $n \times 1$ column matrices, $F(t)$ and $V(t)$ are the two $T$-periodic $m \times m$ matrices defined by

$$
F(t)=\left[\frac{\partial \mathcal{F}_{i}\left(t, x^{*}(t)\right)}{\partial x_{j}}\right]_{1 \leq i, j \leq m}, \quad V(t)=\left[\frac{\partial \mathcal{V}_{i}\left(t, x^{*}(t)\right)}{\partial x_{j}}\right]_{1 \leq i, j \leq m},
$$

$J(t)$ is an $(n-m) \times m$ matrix and $M(t)=\left[\frac{\partial f_{i}\left(t, x^{*}(t)\right)}{\partial x_{j}}\right]_{m+1 \leq i, j \leq n}=-\left[\frac{\partial \mathcal{V}_{i}\left(t, x^{*}(t)\right)}{\partial x_{j}}\right]_{m+1 \leq i, j \leq n}$. Observe also that, in view of $\left(A_{3}\right)$ and $\left(A_{4}\right)$, we have that $F(t)$ is nonnegative and $-V(t)$ is cooperative, that is, the off-diagonal elements of $-V(t)$ are nonnegative.

With these positions, we will assume the following:

$\left(A_{6}\right) \rho\left(\Phi_{-V}\right)<1$, where $\Phi_{-V}$ denotes the monodromy matrix associated to the system $Y^{\prime}=-V(t) Y$ and $\rho(\cdot)$ stands for the spectral radius.

This assumption is natural since it means that the evolution of the first $m$ populations due to deaths and transfers is dissipative and decays exponentially because of deaths.

To complete our preliminary set of hypotheses, we denote by $\mathcal{E} \subseteq \mathbb{R}_{+}^{n}$ the phase space in which system (1) is biologically meaningful and in which we analyze uniform persistence/extinction; in fact, the biological constraints on the variables can imply that the phase space considered is not the whole $\mathbb{R}_{+}^{n}$, and sometimes persistence is not verified for all the initial conditions. Also, for each $\left(x_{0}, s_{0}\right) \in \mathcal{E} \times \mathbb{R}$ we denote by $x\left(t ;\left(x_{0}, s_{0}\right)\right)$ the solution of (1) equal to $x_{0}$ at time $s_{0}$, so that $x\left(s_{0} ;\left(x_{0}, s_{0}\right)\right)=x_{0}$. 
With these preliminaries, our last structural assumption will ask that

$\left(A_{7}\right)$ there exists a bounded set $\mathcal{K} \subset \mathcal{E}$ which is positively invariant and absorbing for the flow of system (1). More precisely, given $s_{0} \in \mathbb{R}$ :

- if $x_{0} \in \mathcal{K}$, we have $x\left(t ;\left(x_{0}, s_{0}\right)\right) \in \mathcal{K}$ for every $t \geq s_{0}$;

- if $x_{0} \in \mathcal{E}$, there exists $t_{1} \geq s_{0}$ such that for every $t \geq t_{1}$ we have $x\left(t ;\left(x_{0}, s_{0}\right)\right) \in \mathcal{K}$.

We now recall the definition of uniform persistence.

Definition 2.1. Under the previous positions, let $1 \leq j \leq n$ be fixed. We say that system (1) is uniformly persistent in $\mathcal{E}$ with respect to $x_{j}$ if there exists $\bar{\delta}>0$ such that, for each initial condition $\left(x_{0}, s_{0}\right) \in \mathcal{E} \times \mathbb{R}$ with $\left(x_{0}\right)_{j}>0$, the solution $x(t)$ of (1) with $x\left(s_{0}\right)=x_{0}$ satisfies

$$
\liminf _{t \rightarrow+\infty} x_{j}(t) \geq \delta
$$

As anticipated in the Introduction, in order to determine whether a system is uniformly persistent with respect to a certain compartment, the role of the basic reproduction number will be essential. In this perspective, we recall that the definition of the basic reproduction number $R_{0}$ for general population models (including PDE ones) in a periodic environment was introduced in [2] and consists in the spectral radius of a linear integral operator on a space of periodic functions (see [3] for an improved presentation). However, in the special case of ODE models, it is possible to alternatively characterize $R_{0}$ through the following lemma [32, Theorem 2.1], which can also serve as a definition.

Lemma 2.2. For every $\lambda>0$, let $\Psi_{\lambda}$ be the monodromy matrix of the system

$$
z^{\prime}=\left(\frac{F(t)}{\lambda}-V(t)\right) z
$$

The following holds:

- if the equation

$$
\rho\left(\Psi_{\lambda}\right)=1
$$

does not have any solution $\lambda>0$, then $R_{0}=0$;

- if there exists a positive solution $\bar{\lambda}$ to $(2)$ (namely, $\rho\left(\Psi_{\bar{\lambda}}\right)=1$ ), then such a solution is unique and $R_{0}:=\bar{\lambda}>0$.

We now state the abstract extinction and persistence results which will be exploited in the next sections. Theorem 2.3 and Proposition 2.4 are slightly different versions of 23 , Theorem 2 and Theorem 4]; we will limit ourselves to giving some comments without providing explicitly the proofs, which are similar to the ones in 23$]$.

Theorem 2.3. Assume $\left(A_{1}\right)$ to $\left(A_{6}\right)$.

1) Assume that $0<R_{0}<1$ and that there exist $\varepsilon^{*}>0$ and $\mu:\left(0, \varepsilon^{*}\right) \rightarrow \mathbb{R}_{+}$such that

i) $\lim _{\varepsilon \rightarrow 0^{+}} \mu(\varepsilon)=1$;

ii) for every $\varepsilon \in\left(0, \varepsilon^{*}\right)$, for any solution $x(t)=\left(x_{1}(t), \ldots, x_{n}(t)\right)^{T}$ of (1) with initial condition $\left(x_{0}, s_{0}\right) \in \mathcal{E} \times \mathbb{R}$, there exists $t_{0} \geq s_{0}$ such that

$$
\tilde{x}^{\prime}(t) \leq\left(\frac{F(t)}{\mu(\varepsilon)}-V(t)\right) \tilde{x}(t), \forall t \geq t_{0},
$$

where $\tilde{x}(t)=\left(x_{1}(t), \ldots, x_{m}(t)\right)^{T}$. 
Then, $\tilde{x}(t) \rightarrow 0$ as $t \rightarrow \infty$. Moreover, if $x^{*}(t)$ is globally asymptotically stable (G.A.S.) in $X_{s} \backslash\{0\}$, then it is G.A.S. in $\mathcal{E}$.

2) Assume that $R_{0}>1$ and that there exists $\tau \in[0, T)$ such that $F(\tau)-V(\tau)$ is irreducible. Fix $j \in\{1, \ldots, m\}$ and assume that $E_{j}:=\left\{x=\left(x_{1}, \ldots, x_{n}\right) \in \mathcal{E} \mid x_{j}=0\right\}$ is closed. Let $\left(A_{7}\right)$ be satisfied for a bounded set $\mathcal{K}$ such that

$$
\emptyset \neq \partial \mathcal{K} \cap E_{j} \subset \mathcal{K} .
$$

Moreover, assume that there exist $\varepsilon^{*}>0$ and $\lambda:\left(0, \varepsilon^{*}\right) \rightarrow \mathbb{R}_{+}$such that:

i) $\lim _{\varepsilon \rightarrow 0^{+}} \lambda(\varepsilon)=1$;

ii) for every $\varepsilon \in\left(0, \varepsilon^{*}\right)$, for any solution $x(t)$ of (1) with initial condition $\left(x_{0}, s_{0}\right) \in \mathcal{K} \times \mathbb{R}$, if there exists $t_{0} \geq s_{0}$ with $x_{j}(t) \leq \varepsilon$ for every $t \geq t_{0}$, then there exists $t_{1} \geq t_{0}$ such that

$$
\tilde{x}^{\prime}(t) \geq\left(\frac{F(t)}{\lambda(\varepsilon)}-V(t)\right) \tilde{x}(t), \forall t \geq t_{1},
$$

where $\tilde{x}(t)=\left(x_{1}(t), \ldots, x_{m}(t)\right)^{T}$.

Then, system 11) is uniformly persistent with respect to $x_{j}$.

Of course, in the previous statement the inequalities (3) and (5) are to be understood component per component. Let us also observe that, contrary to [23, Theorem 2], we do not assume the set $\mathcal{K}$ to be compact. This is due to the fact that the compactness is actually not essential, being there assumed only in order to ensure that

$$
\mathcal{S}=\left\{\left(x_{0}, s_{0}\right) \in \mathcal{K} \times \mathbb{S}^{1} \mid\left(x_{0}\right)_{j}=0\right\}
$$

is compact, with the goal of proving [23, Theorem 2] via the use of [11, Theorem 1]. In our setting, this is obviously guaranteed by (4), which will often be used in the sense that $\mathcal{K}$ contains the "predator-free" part of its boundary.

As a consequence of [23, Theorem 4], we have, on the other hand, the following version of the result on uniform persistence with respect to more compartments.

Proposition 2.4. Assume $\left(A_{1}\right)$ to $\left(A_{6}\right)$. Let $R_{0}>1$ and suppose that there exists $\tau \in[0, T)$ such that $F(\tau)-V(\tau)$ is irreducible. Moreover, assume that $E_{\Sigma}:=\left\{x=\left(x_{1}, \ldots, x_{n}\right) \in \mathcal{E} \mid \sum_{i=1}^{m} x_{i}=0\right\}$ is closed and let $\left(A_{7}\right)$ be satisfied for a bounded set $\mathcal{K}$ such that

$$
\emptyset \neq \partial \mathcal{K} \cap E_{\Sigma} \subset \mathcal{K} \text {. }
$$

Assume finally that there exist $\varepsilon^{*}>0$ and $\lambda:\left(0, \varepsilon^{*}\right) \rightarrow \mathbb{R}_{+}$such that:

i) $\lim _{\varepsilon \rightarrow 0^{+}} \lambda(\varepsilon)=1$;

ii) for every $\varepsilon \in\left(0, \varepsilon^{*}\right)$, for any solution $x(t)$ of (1) with initial condition $\left(x_{0}, s_{0}\right) \in \mathcal{K} \times \mathbb{R}$, if there exists $t_{0} \geq s_{0}$ with $\|\tilde{x}(t)\| \leq \varepsilon$ for every $t \geq t_{0}$, then there exists $t_{1} \geq t_{0}$ such that

$$
\tilde{x}^{\prime}(t) \geq\left(\frac{F(t)}{\lambda(\varepsilon)}-V(t)\right) \tilde{x}(t), \quad \forall t \geq t_{1},
$$

where $\tilde{x}(t)=\left(x_{1}(t), \ldots, x_{m}(t)\right)^{T}$ and again the inequality is meant component per component. Then, system (1) is uniformly persistent with respect to $x_{1}+x_{2}+\cdots+x_{m}$.

Similarly as before, assumption (6) is sufficient in order to ensure the compactness of the set $\left\{x=\left(x_{1}, \ldots, x_{n}\right) \in \mathcal{K} \mid \sum_{i=1}^{m} x_{j}=0\right\}$.

We can apply Theorem 2.3 and Proposition 2.4 to obtain extinction and uniform persistence results for quite general predator-prey systems, as we are going to see in the next section. 


\section{A general one predator-one prey model}

In this section, we consider the following model describing the evolution of a system with one prey and one predator:

$$
\left\{\begin{array}{l}
N^{\prime}=k(t, N) N-f(t, N, P) P \\
P^{\prime}=-\delta_{1}(t) P-\delta_{2}(t) P^{2}+\gamma(t) f(t, N, P) P
\end{array}\right.
$$

and we give a general extinction/uniform persistence result under quite natural requirements on the terms appearing in the model (see Theorem 3.2). This result could be obtained from [26, Theorem $2.2]$; we opted to give a different proof as this is easily generalized to higher dimensions (see Theorem 4.1 and Example 3.7).

We assume that $k: \mathbb{R} \times \mathbb{R}_{+} \rightarrow \mathbb{R}, f: \mathbb{R} \times \mathbb{R}_{+}^{2} \rightarrow \mathbb{R}_{+}, \delta_{i}: \mathbb{R} \rightarrow \mathbb{R}_{+}, i=1,2$, and $\gamma: \mathbb{R} \rightarrow \mathbb{R}_{+}$are continuous functions, $T$-periodic $(T>0)$ in the $t$-variable and continuously differentiable in $N, P$ (if depending on such variables). Here, $k(t, N) N$ corresponds to the growth of the prey in absence of the predators, $f(t, N, P) P$ is the predator functional response and $\gamma(t) f(t, N, P) P$ is the numerical response. The term $\delta_{1}(t)$ corresponds to the death rate of the predators, while $\delta_{2}(t) P^{2}$ is related to an intra-specific competition between predators.

As a preliminary assumption, we ask that

$\delta_{1}(t)>0$ for every $t \in[0, T]$; moreover, if $\delta_{2}(t) \not \equiv 0$, we assume that $\min _{t \in[0, T]} \gamma(t)>0$ (this is actually a quite common assumption in the literature).

We now introduce our main hypotheses on the terms $k(t, N)$ and $f(t, N, P)$. We first deal with the growth of the preys in absence of the predator, assuming the following:

(k) $\left\{\begin{array}{l}k(t, N) \text { is bounded; } \\ k(t, 0)>0, \text { and, for every } t, \text { there exists } N_{1}(t)>0 \text { such that } k\left(t, N_{1}(t)\right)=0 ; \\ \frac{\partial k}{\partial N}<0 \text { for every } N \geq 0 .\end{array}\right.$

Assumption (k) is quite general; for instance, it is satisfied by a logistic growth of the prey population (with $N_{1}(t)$ equal to a constant). Notice that, as a consequence, we have

$$
k(t, N)>0 \text { for } N<N_{1}(t) \quad \text { and } \quad k(t, N)<0 \text { for } N>N_{1}(t) .
$$

Also, we can assume that $N_{1}(t)$ is $T$-periodic.

In the next lemma, we show that, when the predator is absent, such an assumption ensures the existence of a unique periodic solution for the differential equation in (7), and the possibility of continuing such a solution when a small parameter is introduced.

Lemma 3.1. Assume $(\boldsymbol{k})$. Then, there exists $\nu_{*}>0$ such that, for every $0 \leq \nu<\nu_{*}$, there exists a unique positive T-periodic solution $N^{*, \nu}$ to

$$
N^{\prime}=k(t, N) N-\nu N
$$

satisfying

$$
\lim _{\nu \rightarrow 0} N^{*, \nu}(t)=N^{*, 0}(t) \quad \text { uniformly in } t \in[0, T] .
$$

Moreover, for every $\nu \in\left[0, \nu_{*}\left[, N^{*, \nu}(t)\right.\right.$ is globally asymptotically stable. 
Proof. Our proof follows the steps of the proof of [6, Theorem 5]; we will recall in the Appendix some of the abstract results we are going to use throughout it.

We start by making some considerations on equation (9). First, in view of (8) we have that the interval $\left[0, \max _{t \in[0, T]} N_{1}(t)\right]$ acts as an attractor, so that all the solutions to (9) are bounded. Thanks to [14, Theorem 4.11], moreover, it follows that each solution starting with positive initial datum converges to a periodic solution to (9) (possibly an equilibrium). However, solutions starting with (small) positive initial datum cannot converge to zero for $\nu$ sufficiently small; indeed, since $k(t, N)$ is continuous and $k(t, 0)>0$, there exist $\nu^{*}$ and $\bar{N}>0$ such that $k(t, N)-\nu>0$ for every $\nu \in\left[0, \nu^{*}[\right.$, $t \in \mathbb{R}$ and $N \in[0, \bar{N}]$. This implies the existence of a periodic solution $N^{*, \nu}(t)$ for $\nu \in\left[0, \nu^{*}[-\right.$ possibly an equilibrium, satisfying $N^{*, \nu}(t)>\bar{N}$ for every $t$.

Now, let $h(t, N)=k(t, N) N-\nu N$. Since $h(t, 0)=0$ and

$$
h(t, N)-\frac{\partial h(t, N)}{\partial N} N=-N^{2} \frac{\partial k(t, N)}{\partial N}>0 \quad \text { for every } t \in \mathbb{R}, N>0,
$$

by [19, Lemma 4.1 and Theorem 10.2] we have that for each $\nu \in\left[0, \nu^{*}[\right.$ the $T$-Poincaré operator associated with equation $(9)$, which we denote by $P_{\nu}^{T}$, is monotonic, strongly positive and strongly concave. By [19, Theorem 10.1], it follows that the $T$-periodic solution $N^{*, \nu}$ to $(9)$ found above is unique and, by [19, Theorem 10.6 and remark on page 213], it is also globally asymptotically stable. We have finally to prove the validity of 10 . To this end, notice that since the functions $N^{*, \nu}$ satisfy (9) and are bounded, in view of Gronwall's lemma they are uniformly bounded with respect to $\nu$; thus, denoting by $w_{\nu}$ the corresponding fixed point of $P_{\nu}^{T}$, we can assume without loss of generality that $w_{\nu}$ converges. In view of the uniqueness of the fixed point $w_{\nu}$ and the fact that $P_{\nu}^{T}$ depends continuously on $\nu$ when restricted to compact sets, we thus infer that $w_{\nu} \rightarrow w_{0}$, and the assertion (10) follows from the continuous dependence on the initial datum.

In the following, for simplicity, we set $N^{*}=N^{*, 0}$. Also, for each periodic function $z: \mathbb{R} \rightarrow \mathbb{R}_{+}$, we set $\underline{z}=\min _{t \in[0, T]} z(t)$ and $\bar{z}=\max _{t \in[0, T]} z(t)$.

We now turn to the interaction between preys and predators, introducing some conditions on the functional response. In particular, we will always assume the following:

$$
\left\{\begin{array}{l}
f(t, 0, P) \equiv 0 ; \\
\text { fixed } N \text {, the function } P \mapsto f(t, N, P) \text { is nonincreasing in } P \text { (or independent of it); } \\
f(t, N, 0)>0 \text { if } N>0 ; \\
\text { there exists a nonnegative continuous function } f_{0}(t, P) \text { such that it holds } \\
f_{0}(t, P)=\lim _{N \rightarrow 0^{+}} \frac{f(t, N, P)}{N}, \text { uniformly in } t \in \mathbb{R} \text { and } P \text { belonging to compact sets. }
\end{array}\right.
$$

The first two hypotheses essentially say, on one hand, that the predator functional response cannot be positive if there are no preys, and, on the other one, that the more the predators, the less one prey is expected to contribute to their growth. The third and the fourth assumption are very reasonable and simplify the forthcoming proofs.

We now state the main result about (7).

Theorem 3.2. Let $(\boldsymbol{k})$ and $(\boldsymbol{f})$ be satisfied and assume that there exists $\omega>0$ such that the function $N \mapsto f(t, N, P)$ is nondecreasing for $N \leq \bar{N}^{*}+\omega$. Then,

a) the inequality

$$
\int_{0}^{T}\left[\gamma(t) f\left(t, N^{*}(t), 0\right)-\delta_{1}(t)\right] d t<0
$$

implies the extinction of the predators: $P(t) \rightarrow 0$ for $t \rightarrow+\infty$. In this case, we have $\mid N(t)-$ $N^{*}(t) \mid \rightarrow 0$ for $t \rightarrow+\infty$; 
b) the condition

$$
\int_{0}^{T}\left[\gamma(t) f\left(t, N^{*}(t), 0\right)-\delta_{1}(t)\right] d t>0
$$

implies uniform persistence of both $P(t)$ and $N(t)$ for (7).

Remark 3.3. The conditions $(11)$ and 12 compare the per capita rate of growth of the predator class and the total per capita rate of loss of that class near the semi-trivial (stable) periodic solution $\left(N^{*}(t), 0\right)$.

Let us briefly recall some of the most common functional responses for predator-prey models:

$$
f(t, N, P)=m N, \quad(\text { Holling-type I })
$$

$$
f(t, N, P)=\frac{m N}{1+m h N}, \quad \text { (Holling-type II) }
$$

$$
f(t, N, P)=\frac{m N^{2}}{1+m h N^{2}}, \quad(\text { Holling-type III) }
$$

which in its more general version reads as

$$
f(t, N, P)=\frac{m N^{\alpha}}{1+m h N^{\alpha}}, \quad \alpha \geq 1,
$$

as studied by Kazarinov and van den Driessche [18,

$(\mathrm{MH})$

$$
f(t, N, P)=\frac{m N}{a+b N+c N^{2}}, \quad \text { (Monod-Haldane, also called Holling-type IV) }
$$

(BDA)

$$
f(t, N, P)=\frac{m N}{a+b N+c P}, \quad \text { (Beddington-De Angelis) }
$$

$(\mathrm{HV})$

$$
f(t, N, P)=\frac{m N}{a+b N+c P^{\alpha}}, \quad \alpha \in(0,1), \quad \text { (Hassell-Varley) }
$$

$$
f(t, N, P)=\frac{m N}{a+b N+c P+d N P}, \quad \text { (Crowley-Martin) }
$$

(I)

$$
f(t, N, P)=m\left(1-\mathrm{e}^{-c N}\right), \quad(\text { Ivlev })
$$

$$
f(t, N, P)=m\left(1-\mathrm{e}^{-c N / P^{\alpha}}\right), \quad \alpha \in(0,1), \quad(\text { Watt })
$$

where $a, b, c, d, h, m$ can be either positive constants or positive $T$-periodic functions. It can be immediately checked that all these functions satisfy assumption (f), and they are all everywhere increasing in $N$, except for (MH), which is increasing in $N$ as long as $N(t)<\sqrt{a / c}$ (cf. [15]). Hence, we set our result in a quite general scheme which can be adapted to different models. 
Proof of Theorem 3.2. We want to apply Theorem 2.3. We choose $x_{1}=P, x_{2}=N, \mathcal{F}(t, P, N)=$ $(\gamma(t) f(t, N, P) P, 0)^{T}, \mathcal{V}(t, P, N)=\left(\delta_{1}(t) P+\delta_{2}(t) P^{2},-k(t, N) N+f(t, N, P) P\right)^{T}$. With these choices we have

$$
F(t)=\gamma(t) f\left(t, N^{*}(t), 0\right) \quad \text { and } \quad V(t)=\delta_{1}(t),
$$

and assumptions $\left(A_{1}\right)-\left(A_{6}\right)$ are easily satisfied, in view of the requirements on $f(t, N, P), k(t, N)$ and $\delta_{1}(t)$. On the other hand, for technical reasons, we set

$$
\mathcal{E}=\left\{(P, N) \in \mathbb{R}_{+}^{2} \mid \text { if } P>0, \text { then } N>0\right\} .
$$

Notice that this choice does not interfere with the study of extinction. In fact, if we choose an initial condition of the type $(P, 0)$, the corresponding solution converges to $(0,0)$. Also observe that, taking into account [23, Remark 2], if $N(0)>0$ then $N(t)>0$ for each $t>0$ and analogously for the variable $P$.

Proof of part a). In order to prove the first part of the proposition, we apply Theorem 2.3 part 1). Notice that (11) corresponds to having $0<R_{0}<1$ for our model. Now we just have to ensure the existence of a positive function $\mu(\varepsilon)$, defined in a right neighborhood $I$ of $\varepsilon=0$, such that

$$
\lim _{\varepsilon \rightarrow 0^{+}} \mu(\varepsilon)=1
$$

and, for every $\varepsilon \in I, t_{0} \in \mathbb{R},\left(P\left(t_{0}\right), N\left(t_{0}\right)\right) \in \mathcal{E}$, there exists $t_{1} \geq t_{0}$ such that

$$
P^{\prime}(t) \leq\left(\frac{F(t)}{\mu(\varepsilon)}-V(t)\right) P(t)
$$

for every $t \geq t_{1}$ (condition 1)-ii) of Theorem 2.3p. To this end, we make a comparison between the solutions of the first differential equation in (7) and the solutions $\bar{N}(t)$ to

$$
\bar{N}^{\prime}=k(t, \bar{N}) \bar{N}
$$

satisfying the same initial condition. On one hand, fixed an initial condition we clearly have that the solution to (13) is an upper solution; on the other hand, Lemma 3.1 and the fact that $N(t) \equiv 0$ is a lower solution ensure that, for $\varepsilon>0$ fixed, there exists $t_{1} \geq t_{0}$ such that

$$
0 \leq N(t) \leq \bar{N}(t) \leq N^{*}(t)+\varepsilon,
$$

for every $t \geq t_{1}$. From the fact that $N \mapsto f(t, N, P)$ is nondecreasing (we can assume that $\varepsilon<\omega$ ) and using (f), we can now infer

$$
\begin{aligned}
P^{\prime} & =-\delta_{1}(t) P-\delta_{2}(t) P^{2}+\gamma(t) f(t, N, P) P \leq-\delta_{1}(t) P+\gamma(t) f\left(t, N^{*}(t)+\varepsilon, P\right) P \\
& \leq-\delta_{1}(t) P+\gamma(t) f\left(t, N^{*}(t)+\varepsilon, 0\right) P .
\end{aligned}
$$

We can thus choose

$$
\mu(\varepsilon)=\min _{t \in[0, T]} \frac{f\left(t, N^{*}(t), 0\right)}{f\left(t, N^{*}(t)+\varepsilon, 0\right)}
$$

and the claim is proved.

Proof of part b). In order to prove the second part of the proposition, corresponding to the uniform persistence result, we now want to apply Theorem 2.3 part 2). Notice preliminarily that (12) corresponds to stating that the basic reproduction number $R_{0}$ for system $(7)$ is greater than 1 .

We first construct a bounded invariant attractor $\mathcal{K}$ fulfilling $\left(A_{7}\right)$ and (4). To this end, focusing first on the prey, we see that if $N\left(t_{0}\right) \geq 0$ is the initial value for the prey population, then $N(t) \geq 0$ for every $t \geq t_{0}$. On the other hand, as already seen in the proof of part a), the solution to $(13)$ is an 
upper solution for the first equation in (7), thus giving an upper bound for it. Reasoning as for (14), we can thus infer that, fixed a small number $\kappa>0$, there exists $t_{1}>t_{0}$ such that

$$
0 \leq N(t) \leq \bar{N}(t) \leq \bar{N}^{*}+\kappa \leq \mathfrak{N}:=\bar{N}_{1}+\kappa
$$

(where $N_{1}(t)$ has been defined in assumption $(\mathbf{k})$ ), for every $t \geq t_{1}$. It follows that every solution $N(t)$ is bounded and it is clear that if $N\left(t_{0}\right) \leq \mathfrak{N}$, then $N(t) \leq \mathfrak{N}$ for every $t \geq t_{0}$. Hence, looking only at the prey, $\{0 \leq N \leq \mathfrak{N}\}$ is an invariant attractor.

Let us turn to the predator, observing preliminarily that if $P(0) \geq 0$, then $P(t) \geq 0$ for every $t \in[0, T]$. Set now $\bar{k}=\max _{t \in[0, T], N \in[0, \mathfrak{N}]} k(t, N)$. We have

$$
\begin{aligned}
(\bar{\gamma} N+P)^{\prime} & =\bar{\gamma}(k(t, N) N-f(t, N, P) P)-\delta_{1}(t) P-\delta_{2}(t) P^{2}+\gamma(t) f(t, N, P) P \\
& \leq \bar{\gamma} k(t, N) N-\underline{\delta} P \leq \bar{\gamma} \mathfrak{N}(\bar{k}+\underline{\delta})-\underline{\delta}(\bar{\gamma} N+P) .
\end{aligned}
$$

Consequently, there exists a suitable $t_{1}>t_{0}$ such that, for every $t \geq t_{1}$,

$$
\bar{\gamma} N(t)+P(t) \leq 2 \frac{\bar{\gamma} \mathfrak{N}(\bar{k}+\underline{\delta})}{\underline{\delta}}=: \mathfrak{S} .
$$

Moreover, if $N\left(t_{0}\right) \leq \mathfrak{N}$ (so that $N(t) \leq \mathfrak{N}$ for every $t \geq t_{0}$ ) and $\bar{\gamma} N\left(t_{0}\right)+P\left(t_{0}\right) \leq \mathfrak{S}$, then 15 holds for every $t \geq t_{0}$. We can thus define a first invariant attractor

$$
\mathcal{K}^{\prime}=\left\{(P, N) \in \mathbb{R}_{+}^{2} \mid P \geq 0,0 \leq N \leq \mathfrak{N}, \bar{\gamma} N+P \leq \mathfrak{S}\right\} .
$$

However, for our purposes we need a small modification of $\mathcal{K}^{\prime}$, in order to avoid the trivial dynamics which appears starting with no preys. Noticing that, as we previously said, the axes are invariant and that initial conditions outside the axes give rise to strictly positive solutions, (namely, if $(P(0), N(0)) \in \operatorname{int} \mathbb{R}_{+}^{2}$, then $(P(t), N(t)) \in \operatorname{int} \mathbb{R}_{+}^{2}$ for every $\left.t \geq 0\right)$, we thus set

$$
\mathcal{K}=\mathcal{K}^{\prime} \cap \mathcal{E} .
$$

Such a set is an attractor of all the orbits starting in $\mathcal{E}$ and fulfills (4) (with $x_{j}=P$ ), as it is easily seen.

To show uniform persistence with respect to $P$, we now aim at proving that there exists a positive function $\lambda(\varepsilon)$, defined in a right neighborhood $I$ of $\varepsilon=0$, such that

$$
\lim _{\varepsilon \rightarrow 0^{+}} \lambda(\varepsilon)=1
$$

and, for every $\varepsilon \in I$, if $t_{0}>0$ is such that $P(t) \leq \varepsilon$ for every $t \geq t_{0}$, then there exists $t_{1} \geq t_{0}$ such that

$$
P^{\prime}(t) \geq\left(\frac{F(t)}{\lambda(\varepsilon)}-V(t)\right) P(t)
$$

for every $t \geq t_{1}$ (condition 2)-ii) of Theorem 2.3, provided that the initial condition is chosen in $\mathcal{K}$. We preliminarily observe that, in case $P(0)=0$, we have $P(t)=0$ for every $t \in[0, T]$ and the dynamics is trivial; in particular, inequality (16) is trivially satisfied, since both sides vanish. Moreover, in view of the definitions of $F(t)$ and $V(t), \sqrt{16})$ is immediately satisfied also for the time instants $\bar{t}$ (if any) for which $\gamma(\bar{t})=0$ (and so $F(\bar{t})=0$; if this happens at least once, then $\delta_{2}(t) \equiv 0$ in view of the hypotheses). For this reason, we can take into account only the time instants $t$ such that $\gamma(t)>0$ and assume that the initial condition is chosen in int $\mathbb{R}_{+}^{2}$, being in particular $N(0)>0$. Recall that, once $N(0)$ is fixed, $N(t)$ is uniquely determined in view of the Cauchy Theorem.

We first notice that there exists $C_{*}>0$ such that, for every $\left(P\left(t_{0}\right), N\left(t_{0}\right)\right) \in \mathcal{K}^{\prime}\left(t_{0} \in \mathbb{R}\right)$, it holds $f(t, N, P) \leq C_{*} N$ for every $t \geq t_{0}$. This follows from the fact that $N$ and $P$ lie in compact sets (in view of the invariance of $\mathcal{K}^{\prime}$ ) and from the continuity of $f_{0}(t, P)$. Let us now fix 
$0<\varepsilon_{1}<\min \left\{\nu_{*} / C_{*}, \omega\right\}$ and set, for each $0<\varepsilon<\varepsilon_{1}, \nu(\varepsilon)=C_{*} \varepsilon$. If there exists $t_{0} \in \mathbb{R}$ such that $P(t) \leq \varepsilon$ for each $t \geq t_{0}$, we thus have that

$$
k(t, N) N-\nu(\varepsilon) N \leq N^{\prime} \leq k(t, N) N, \quad \forall t \geq t_{0} .
$$

From Lemma 3.1. we conclude that for each $\delta>0$ there exists $t_{*}(\delta) \geq t_{0}$ such that $N^{*, \nu(\varepsilon)}(t)-\delta \leq$ $N(t) \leq N^{*}(t)+\delta$ for each $t \geq t_{*}(\delta)$.

At this point, choose $\delta=\varepsilon$; by the monotonicity of $f(t, N, P)$ in the $N$-variable for $N \leq \bar{N}^{*}+\omega$, we immediately infer the existence of $t_{1}=t_{*}(\varepsilon)$ such that if $t \geq t_{1}$ we have

$$
f(t, N, P) \geq f(t, N, \varepsilon) \geq f\left(t, N^{*, \nu(\varepsilon)}-\varepsilon, \varepsilon\right) .
$$

Setting $\lambda(\varepsilon)=\max _{t \in[0, T]} \frac{f\left(t, N^{*}(t), 0\right)}{f\left(t, N^{*, \nu}(\varepsilon)(t)-\varepsilon, \varepsilon\right)-\delta_{2}(t) \varepsilon}$, the inequality 16 is satisfied and the uniform persistence of $P$ follows.

Having proved that the system is uniformly persistent with respect to $P$, the uniform persistence of $N$ easily follows from [11, Theorem 1], which allows to deduce that $\{N=0\}$ is a uniform repeller.

For completeness, let us say that, once persistence of $P$ has been obtained in the previous proof, we can deduce the uniform persistence of $N$ using another simple reasoning, which we briefly show. First of all, as a consequence of the uniform persistence of $P$, there exists $\delta>0$ such that $\limsup _{t \rightarrow+\infty} N(t)>\delta$. In fact, as $f(t, N, P) \leq f(t, N, 0)$ for every $P \geq 0$ and $f(t, 0,0)=0$, there exists $\delta>0$ such that if $0 \leq N \leq \delta$, then

$$
\max _{t \in[0, T], N \in[0, \delta], P \in \mathbb{R}_{+}} f(t, N, P)<\underline{\delta} / 2 \bar{\gamma} .
$$

Then, if it were $\lim \sup _{t \rightarrow+\infty} N(t) \leq \delta$, from the second differential equation in (7) we would obtain $P(t) \rightarrow 0$ as $t \rightarrow+\infty$, contradicting the uniform persistence of $P$. This means that $N$ is weakly uniformly persistent and hence, by [35, Theorem 1.3.3], we have that the system is uniformly persistent.

As a consequence of the persistence of both the predator and the prey if $R_{0}>1$, we have the following.

Proposition 3.4. Under the assumptions of Theorem 3.2, part b), there exists a nontrivial $T$ periodic solution $(N(t), P(t))$ to (7) such that $N(t), P(t)>0$ for every $t \in[0, T]$.

Proof. The statement follows from [35, Theorem 1.3.6].

As an example, Theorem 3.2 and Proposition 3.4 above apply to the following predator-prey model with Beddington-De Angelis functional response, proposed in [8]:

$$
\left\{\begin{array}{l}
x^{\prime}=x\left[a(t)-b(t) x-\frac{c(t) y}{\alpha(t)+\beta(t) x+\gamma(t) y}\right] \\
y^{\prime}=y\left[-d(t)+\frac{f(t) x}{\alpha(t)+\beta(t) x+\gamma(t) y}\right]
\end{array}\right.
$$

where $a(\cdot), b(\cdot), c(\cdot), d(\cdot), f(\cdot), \alpha(\cdot), \beta(\cdot), \gamma(\cdot)$ are $T$-periodic continuous and positive functions. In 8 , the authors only assume that the mean of $a(\cdot)$ is positive and that $b(\cdot)$ is nonnegative, but not identically equal to zero; however, we can also assume this more general condition if we adapt Lemma 3.1 to this situation. As in that paper, our general results imply uniform persistence and the 
existence of a positive periodic orbit (resp. extinction) if $\int_{0}^{T}-d(t)+\frac{f(t) x^{*}(t)}{\alpha(t)+\beta(t) x^{*}(t)} d t>0$ (resp. $<0$ ); here, as usual, we denote by $x^{*}(t)$ the periodic solution in the predator-free space. We stress that, in this case, a functional response of type (HV) would produce the same outcome.

Remark 3.5. The situation described in the previous results can be extended to models with more preys and one predator, provided that an analogue of Lemma 3.1 can be proved, stating in particular the existence of a globally asymptotically stable predator-free periodic orbit. For instance, assuming a logistic growth for both the preys, it is possible to prove the following:

Proposition 3.6. Assume that $a_{i}: \mathbb{R} \rightarrow \mathbb{R}_{+}, i=1,2$, are positive $T$-periodic functions. For every $0 \leq \nu<1$, there exists a unique T-periodic orbit for the system

$$
\left\{\begin{array}{l}
N_{1}^{\prime}=N_{1}\left(1-\frac{N_{1}}{K_{1}}\right)+a_{1}(t) N_{2}-a_{2}(t) N_{1}-\nu N_{1} \\
N_{2}^{\prime}=N_{2}\left(1-\frac{N_{2}}{K_{2}}\right)-a_{1}(t) N_{2}+a_{2}(t) N_{1}-\nu N_{2}
\end{array}\right.
$$

that we denote by $0 \not \equiv\left(N_{1}^{*, \nu}(t), N_{2}^{*, \nu}(t)\right) \in \mathbb{R}_{+}^{2}$, which attracts all the orbits of 18 such that $N_{1}(0)>0, N_{2}(0)>0$. Moreover,

$$
\lim _{\nu \rightarrow 0}\left(N_{1}^{*, \nu}(t), N_{2}^{*, \nu}(t)\right)=\left(N_{1}^{*}(t), N_{2}^{*}(t)\right), \quad \text { uniformly in } t \in[0, T],
$$

where we denote by $\left(N_{1}^{*}(t), N_{2}^{*}(t)\right)$ the solution to 18$)$ with $\nu=0$.

Proof. Our proof follows again the steps of the proof of [6. Theorem 5].

First of all, summing the two differential equations in 180 we see that there exist $K_{1}, K_{2}>0$ such that $y=N_{1}+N_{2}$ satisfies $y\left(1-\nu-\frac{y}{K_{1}}\right) \leq y^{\prime} \leq y\left(1-\frac{y}{K_{2}}\right)$. Hence, for every sufficiently large $t$, we have that $N_{1}+N_{2}>0$ implies $\frac{K_{1}}{2}(1-\nu) \leq N_{1}+N_{2} \leq 2 K_{2}$. From this, it is immediate to conclude that there exists a compact attractor $\hat{\mathcal{K}}$ of the orbits of (18); we now want to show that $\hat{\mathcal{K}}$ is contained in the set where $N_{1}>0$ and $N_{2}>0$. However, this is ensured by the fact that $N_{1}$ and $N_{2}$ are uniformly persistent, as it is possible to prove, for example, using [11, Theorem 1]. As a consequence, we can apply Massera's theorem and conclude the existence of a positive periodic orbit of the system for each $0 \leq \nu<1$.

In order to prove the remaining part of the proposition, let us now define

$$
\left\{\begin{array}{l}
h_{1}\left(t, N_{1}, N_{2}\right):=N_{1}\left(1-\frac{N_{1}}{K_{1}}\right)+a_{1}(t) N_{2}-a_{2}(t) N_{1}-\nu N_{1} \\
h_{2}\left(t, N_{1}, N_{2}\right):=N_{2}\left(1-\frac{N_{2}}{K_{2}}\right)-a_{1}(t) N_{2}+a_{2}(t) N_{1}-\nu N_{2} .
\end{array}\right.
$$

We have that $h_{1}\left(t, 0, N_{2}\right)=a_{1}(t) N_{2} \geq 0$ and $h_{2}\left(t, N_{1}, 0\right)=a_{2}(t) N_{1} \geq 0$ for every $t \in \mathbb{R}$ and every $\left(N_{1}, N_{2}\right) \in \mathbb{R}_{+}^{2}$ with $N_{1}+N_{2}>0$; moreover, $h_{i}\left(t, N_{1}, N_{2}\right)-\sum_{j=1}^{2} \frac{\partial h_{i}\left(t, N_{1}, N_{2}\right)}{\partial N_{j}} N_{j}>0$ for every $t \in \mathbb{R}$ and every $\left(N_{1}, N_{2}\right) \in \mathbb{R}_{+}^{2}$ with $N_{1}>0, N_{2}>0$. The result now follows as in the proof of Lemma 3.1 .

Example 3.7. As an example, in [15] the following predator-prey model with Monod-Haldane 
functional response was considered:

$$
\left\{\begin{array}{l}
x_{1}^{\prime}=x_{1}\left[b_{1}(t)-a_{1}(t) x_{1}-\frac{c_{1}(t) y}{e(t)+\beta(t) x_{1}+x_{1}^{2}}\right]+D(t)\left(x_{2}-x_{1}\right) \\
x_{2}^{\prime}=x_{2}\left(b_{2}(t)-a_{2}(t) x_{2}\right)+D(t)\left(x_{1}-x_{2}\right) \\
y^{\prime}=y\left[-d(t)+\frac{c_{2}(t) x_{1}}{e(t)+\beta(t) x_{1}+x_{1}^{2}}-q(t) y\right],
\end{array}\right.
$$

where, keeping the same notation therein, $x_{1}$ and $x_{2}$ are the preys and $y$ is the predator. In such a model, as usual, the functions $a_{i}(\cdot), b_{i}(\cdot), c_{i}(\cdot), d(\cdot), e(\cdot), \beta(\cdot), q(\cdot)$ and $D(\cdot)$ are all positive, continuous and $T$-periodic. Our general results, as stated, give conditions for extinction and for uniform persistence if $x_{1}^{*}(t)<\sqrt{e(t)}$ (condition which guarantees that $f(t, N, P)$ is nondecreasing in $N)$. In 15, the same results on extinction/persistence were obtained under the same assumption $x_{1}^{*}(t)<\sqrt{e(t)}$, but the case $R_{0}=1$, which is not included in our framework, was also analyzed.

As a final remark, let us show explicitly that for this model, speaking about persistence, it is sufficient to perform a direct computation in order to apply Theorem 2.3. also without the monotonicity assumption. To this end, recall that here we have

$$
F(t)=\frac{c_{2} N_{1}^{*}(t)}{e(t)+\beta(t) N_{1}^{*}(t)+N_{1}^{*}(t)^{2}}, \quad V(t)=d(t) .
$$

Thus, wishing to apply part 2) of Theorem 2.3 , fixed $\varepsilon_{1}>0$ and $\nu(\varepsilon)$ as in the proof of Theorem 3.2 assume that $y(t) \leq \varepsilon<\varepsilon_{1}$ for every $t \geq t_{0}$. Thanks to Proposition 3.6 and an analogue of (17), for a suitable $t_{1}(\varepsilon) \geq t_{0}$ we have

$$
N_{1}^{*, \nu(\varepsilon)}(t)-\varepsilon \leq x_{1}(t) \leq N_{1}^{*}(t)
$$

so that, for $t \geq t_{1}(\varepsilon)$,

$y^{\prime}=y\left[-d(t)+\frac{c_{2}(t) x_{1}}{e(t)+\beta(t) x_{1}+x_{1}^{2}}-q(t) y\right] \geq y\left[-d(t)-q(t) \varepsilon+\frac{c_{2}(t) N_{1}^{*, \nu}(\varepsilon)(t)-c_{2}(t) \varepsilon}{e(t)+\beta(t) N_{1}^{*}(t)+N_{1}^{*}(t)^{2}}\right]$.

Hence, we obtain that $y^{\prime} \geq\left(\frac{F(t)}{\lambda(\varepsilon)}-V(t)\right) y$ for the choice

$$
\lambda(\varepsilon)=\max _{t \in[0, T]} \frac{c_{2} N_{1}^{*}(t)}{c_{2} N_{1}^{*, \nu(\varepsilon)}(t)-c_{2}(t) \varepsilon-q(t) \varepsilon\left[e(t)+\beta(t) N_{1}^{*}(t)+N_{1}^{*}(t)^{2}\right]} .
$$

Being $\lambda(\varepsilon) \rightarrow 1$ for $\varepsilon \rightarrow 0$, all the assumptions of Theorem 2.3 are satisfied. This shows that Theorem 2.3 may be applicable even if not all the hypotheses of Theorem 3.2 are fulfilled, by performing direct computations on the considered model; hence, when the monotonicity is not fulfilled the models might possibly be studied directly, case by case. It is clear that this reasoning may work also for very general kinds of functional responses.

Wishing, on the other hand, to consider a model with more predators, the situation is a little bit more complicated, as we are going to see in the following section.

\section{Models with more than one predator}

In this section, we present possible extensions of Theorem 3.2 to situations where we take into account the presence of more predators. For simplicity, we will restrict ourselves to consider systems with only one prey; of course, other generalizations would be possible, at the expense of a possibly heavier formulation (see Remark 4.7 below). 
Let us first consider a model in which the predators $P_{1}, P_{2}$ hunt the prey $N$ without interacting one with the other:

$$
\left\{\begin{array}{l}
N^{\prime}=k(t, N) N-f\left(t, N, P_{1}\right) P_{1}-g\left(t, N, P_{2}\right) P_{2} \\
P_{1}^{\prime}=-\xi_{1}(t) P_{1}-\eta_{1}(t) P_{1}^{2}+\alpha(t) f\left(t, N, P_{1}\right) P_{1} \\
P_{2}^{\prime}=-\xi_{2}(t) P_{2}-\eta_{2}(t) P_{2}^{2}+\beta(t) g\left(t, N, P_{2}\right) P_{2} .
\end{array}\right.
$$

Here $k: \mathbb{R} \times \mathbb{R}_{+} \rightarrow \mathbb{R}$ is continuous and $T$-periodic in the $t$-variable and differentiable in $N$. We also assume that $f, g: \mathbb{R} \times \mathbb{R}_{+}^{2} \rightarrow \mathbb{R}_{+}, \alpha, \beta, \xi_{i}, \eta_{i}: \mathbb{R} \rightarrow \mathbb{R}_{+}, i=1,2$, are continuous, $T$-periodic in the $t$-variable and differentiable in the other variables (if depending explicitly on them). For simplicity, we also suppose that $\alpha(t), \beta(t)>0$ for every $t$, recalling that, similarly as in the previous section, it would be possible to let them vanish somewhere, assuming for instance that the corresponding $\eta_{i}$ is identically zero.

Under this framework, denoting by $N^{*}(t)$ the unique nontrivial $T$-periodic solution to $N^{\prime}=k(t, N) N$ (see Lemma 3.1), we have the following statement.

Theorem 4.1. Assume the following hypotheses:

- the function $k(t, N)$ satisfies assumption $(\boldsymbol{k})$;

- the functions $f(t, N, P)$ and $g(t, N, P)$ satisfy assumption $(\boldsymbol{f})$;

- $\xi_{1}(t), \xi_{2}(t)>0$ for every $t \in[0, T]$;

- there exists $\omega>0$ such that the functions $N \mapsto f(t, N, P), N \mapsto g(t, N, P)$ are nondecreasing for $N \leq \bar{N}^{*}+\omega$.

Then,

a) the inequalities

$$
\frac{\int_{0}^{T} \alpha(t) f\left(t, N^{*}(t), 0\right) d t}{\int_{0}^{T} \xi_{1}(t) d t}<1 \quad \text { and } \quad \frac{\int_{0}^{T} \beta(t) g\left(t, N^{*}(t), 0\right) d t}{\int_{0}^{T} \xi_{2}(t) d t}<1
$$

imply the extinction of both the predators for (7), that is, $P_{i}(t) \rightarrow 0$ for $t \rightarrow+\infty, i=1,2$;

b) the condition

$$
\frac{\int_{0}^{T} \alpha(t) f\left(t, N^{*}(t), 0\right) d t}{\int_{0}^{T} \xi_{1}(t) d t}>1 \quad \text { or } \quad \frac{\int_{0}^{T} \beta(t) g\left(t, N^{*}(t), 0\right) d t}{\int_{0}^{T} \xi_{2}(t) d t}>1
$$

implies the uniform persistence of $P_{1}+P_{2}$ for (7).

Proof. Similarly as in Theorem 3.2 , we choose $x_{1}=P_{1}, x_{2}=P_{2}, x_{3}=N$ and we set $\mathcal{F}\left(t, P_{1}, P_{2}, N\right)=$ $\left(\alpha(t) f\left(t, N, P_{1}\right) P_{1}, \beta(t) g\left(t, N, P_{2}\right) P_{2}, 0\right)^{T}, \mathcal{V}\left(t, P_{1}, P_{2}, N\right)=\left(\xi_{1}(t) P_{1}+\eta_{1}(t) P_{1}^{2}, \xi_{2}(t) P_{2}+\eta_{2}(t) P_{2}^{2},-k(t, N) N+\right.$ $\left.f\left(t, N, P_{1}\right) P_{1}+g\left(t, N, P_{2}\right) P_{2}\right)^{T}$. With these choices, we have

$$
F(t)=\left[\begin{array}{cc}
\alpha(t) f\left(t, N^{*}(t), 0\right) & 0 \\
0 & \beta(t) g\left(t, N^{*}(t), 0\right)
\end{array}\right],
$$

and

$$
V(t)=\left[\begin{array}{cc}
\xi_{1}(t) & 0 \\
0 & \xi_{2}(t)
\end{array}\right]
$$

and it can be checked without difficulties that assumptions $\left(A_{1}\right)-\left(A_{6}\right)$ in Theorem 2.3 are satisfied. 
Proof of part a). To prove the extinction result, we apply Theorem 2.3, part 1). It is immediately checked that (20) corresponds to $R_{0}<1$. Defining

$$
\mathcal{E}=\left\{\left(P_{1}, P_{2}, N\right) \in \mathbb{R}_{+}^{3} \mid \text { if } P_{1}^{2}+P_{2}^{2}>0, \text { then } N>0\right\}
$$

we now want to ensure the existence of a positive function $\mu(\varepsilon)$, defined in a neighborhood $I$ of $\varepsilon=0$, such that

$$
\lim _{\varepsilon \rightarrow 0^{+}} \mu(\varepsilon)=1
$$

and, for every $\varepsilon \in I, t_{0} \in \mathbb{R}$ and $\left(P_{1}(0), P_{2}(0), N(0)\right) \in \mathcal{E}$, there exists $t_{1} \geq t_{0}$ such that

$$
P^{\prime}(t) \leq\left(\frac{F(t)}{\mu(\varepsilon)}-V(t)\right) P(t)
$$

for every $t \geq t_{1}$, where $P(t)=\left(P_{1}(t), P_{2}(t)\right)$. However, reasoning similarly as in the proof of Theorem 3.2, we have that $N(t) \geq 0$ for every $t$, and using Lemma 3.1 we deduce that, for every fixed $\varepsilon>0$, there exists $t_{1} \geq t_{0}$ such that

$$
0 \leq N(t) \leq N^{*}(t)+\varepsilon,
$$

for every $t \geq t_{1}$. Thanks to the fact that $f(t, N, P)$ is nondecreasing in $N$ and using (f), we can now infer

$$
P_{1}^{\prime}(t)=-\xi_{1}(t) P_{1}-\eta_{1}(t) P_{1}^{2}+\alpha(t) f\left(t, N, P_{1}\right) P_{1} \leq-\xi_{1}(t) P_{1}+\alpha(t) f\left(t, N^{*}+\varepsilon, 0\right) P_{1},
$$

and a similar estimate holds for $P_{2}$. Setting

$$
\mu_{1}(\varepsilon)=\min _{t \in[0, T]} \frac{f\left(t, N^{*}(t), 0\right)}{f\left(t, N^{*}(t)+\varepsilon, 0\right)}, \quad \mu_{2}(\varepsilon)=\min _{t \in[0, T]} \frac{g\left(t, N^{*}(t), 0\right)}{g\left(t, N^{*}(t)+\varepsilon, 0\right)},
$$

the conclusion then follows by defining

$$
\mu(\varepsilon)=\min \left\{\mu_{1}(\varepsilon), \mu_{2}(\varepsilon)\right\}
$$

Proof of part b). In order to prove the uniform persistence result, we want to apply Proposition 2.4 We first check the validity of $\left(A_{7}\right)$. Set

$$
A:=\max \left\{\max _{t \in[0, T]} \alpha(t), \max _{t \in[0, T]} \beta(t)\right\},
$$

$B:=\min _{i=1,2} \min _{t \in[0, T]} \xi_{i}(t)>0, K=\max _{t \in[0, T], N \in \mathbb{R}} k(t, N)$ and observe that

$$
\begin{aligned}
\left(A N+P_{1}+P_{2}\right)^{\prime} & \leq K A N-\xi_{1} P_{1}-\xi_{2} P_{2} \leq(B+K) A N-B\left(A N+P_{1}+P_{2}\right) \\
& \leq \Gamma-B\left(A N+P_{1}+P_{2}\right),
\end{aligned}
$$

where $\Gamma:=A(B+K) \mathcal{N}<+\infty$, having denoted by $\mathcal{N}$ a suitable upper bound for $N(t)$, which is finite in view of assumption (k). Thus, in view of [23, Lemma 1], we can define a first invariant compact attractor

$$
\mathcal{K}^{\prime}=\left\{\left(P_{1}, P_{2}, N\right) \in \mathbb{R}_{+}^{3} \mid A N+P_{1}+P_{2} \leq \frac{2 \Gamma}{B}\right\}
$$

One can thus reason analogously as in the proof of Theorem 3.2 to obtain the bounded set required in condition $\left(A_{7}\right)$, by setting

$$
\mathcal{K}:=\mathcal{K}^{\prime} \cap \mathcal{E}
$$

After having noticed that (21) implies $R_{0}>1$, we now aim at proving that there exists a positive function $\lambda(\varepsilon)$, defined in a neighborhood $I$ of $\varepsilon=0$, such that

$$
\lim _{\varepsilon \rightarrow 0^{+}} \lambda(\varepsilon)=1
$$


and, for every $\varepsilon \in I$, if $t_{0}>0$ is such that $\left\|\left(P_{1}(t), P_{2}(t)\right)\right\| \leq \varepsilon$ for every $t \geq t_{0}$, then there exists $t_{1} \geq t_{0}$ such that

$$
P^{\prime}(t) \geq\left(\frac{F(t)}{\lambda(\varepsilon)}-V(t)\right) P(t)
$$

for every $t \geq t_{1}$ (where $P(t)=\left(P_{1}(t), P_{2}(t)\right)$ and the inequality is understood component per component), provided that the initial condition is chosen in $\mathcal{K}$. In this way, condition ii) in Proposition 2.4 will be fulfilled.

We first observe that, in case $\left(P_{1}(0), P_{2}(0)\right)=(0,0)$, we have $\left(P_{1}(t), P_{2}(t)\right)=(0,0)$ for every $t \in[0, T]$ and the dynamics is trivial; in particular, inequality $(22)$ is trivially satisfied. For this reason, we can assume without loss of generality that the initial condition is chosen in int $\mathbb{R}_{+}^{3}$.

The proof now proceeds as for Theorem 3.2. after having set, for some continuous functions $\nu_{i}(\varepsilon)$ such that $\lim _{\varepsilon \rightarrow 0^{+}} \nu_{i}(\varepsilon)=0, i=1,2$,

$$
\lambda_{1}(\varepsilon)=\max _{t \in[0, T]} \frac{f\left(t, N^{*}(t), 0\right)}{f\left(t, N^{*}, \nu_{1}(\varepsilon)(t)-\varepsilon, \varepsilon\right)-\eta_{1}(t) \varepsilon},
$$

and

$$
\lambda_{2}(\varepsilon)=\max _{t \in[0, T]} \frac{g\left(t, N^{*}(t), 0\right)}{g\left(t, N^{*, \nu_{2}(\varepsilon)}(t)-\varepsilon, \varepsilon\right)-\eta_{2}(t) \varepsilon} .
$$

Indeed, the remaining part of the argument requires only slight changes with respect to Theorem 3.2 and is left to the reader, taking into account that the final function $\lambda(\varepsilon)$ will be defined as

$$
\lambda(\varepsilon)=\max \left\{\lambda_{1}(\varepsilon), \lambda_{2}(\varepsilon)\right\} .
$$

Similarly as for the one prey-one predator case, we can deduce a corollary about the existence of a nontrivial $T$-periodic solution.

Proposition 4.2. Under the assumptions of Theorem 4.1, there exists a nontrivial T-periodic solution $\left(N(t), P_{1}(t), P_{2}(t)\right)$ to $19 p$ such that $P_{1}(t)+P_{2}(t)>0$ for every $t \in[0, T]$.

Proof. The statement follows from [35, Theorem 1.3.6].

Of course, in case we are able to guarantee the uniform persistence of each predator, [27, Theorem 6.2] ensures the existence of a $T$-periodic solution which does not touch $\partial \mathcal{K}$.

Remark 4.3. Analogously to what was done in 24, we can deal in a similar manner with models having a delay. In this case, however, we do not have an explicit formula for $R_{0}$.

We now briefly explore some other models in which persistence can be proved analogously.

1) We can admit an interaction between the two predators, both of competitive (which seems to be biologically more significant, speaking about predators) and of cooperative nature, namely considering a model like

$$
\left\{\begin{array}{l}
N^{\prime}=k(t, N) N-f\left(t, N, P_{1}\right) P_{1}-g\left(t, N, P_{2}\right) P_{2} \\
P_{1}^{\prime}=-\xi_{1}(t) P_{1}-\eta_{1}(t) P_{1}^{2}+\alpha(t) f\left(t, N, P_{1}\right) P_{1}+\gamma_{1}(t) P_{1} P_{2} \\
P_{2}^{\prime}=-\xi_{2}(t) P_{2}-\eta_{2}(t) P_{2}^{2}+\beta(t) g\left(t, N, P_{2}\right) P_{2}+\gamma_{2}(t) P_{1} P_{2},
\end{array}\right.
$$

where $\gamma_{1}, \gamma_{2}: \mathbb{R} \rightarrow \mathbb{R}$ are continuous and $T$-periodic functions. In this case, however, if the interaction is cooperative (namely $\gamma_{i} \geq 0$ for $i=1,2$ ), we will assume for the sake of simplicity that $n:=\min _{i=1,2} \min _{t \in[0, T]} \eta_{i}(t)$ is strictly positive and such that $\gamma \leq n$, where 
$\gamma:=\max _{i=1,2} \max _{t \in[0, T]} \gamma_{i}(t)$. This is done in order to ensure that $2 \gamma P_{1} P_{2}-n P_{1}^{2}-n P_{2}^{2} \leq 0$, so that, wishing to find the attractor $\mathcal{K}$, a similar estimate as before for $\left(A N+P_{1}+P_{2}\right)^{\prime}$ can be obtained.

On the other hand, the matrices $F(t)$ and $V(t)$ do not change with respect to Theorem 4.1 , since the linearizations are computed in the predators-free state. Wishing to prove the validity of condition ii) in Proposition 2.4 when $\left\|\left(P_{1}, P_{2}\right)\right\| \leq \epsilon$, we now have to control from below the part due to the interaction between the predators. When estimating $P_{i}^{\prime}(i=1$ or $i=2)$, the control is given by 0 if $\gamma_{i}$ is positive, or by the inequality

$$
\gamma_{i}(t) P_{i} P_{j} \geq \epsilon \gamma_{i}(t) P_{i}, \quad i \neq j, \quad i, j=1,2
$$

if $\gamma_{i}$ is negative. It is not difficult, then, to reason as before to obtain the following proposition.

Proposition 4.4. Let $R_{0}$ be the basic reproduction number for 23, defined as in Section 2. Then,

- if $R_{0}<1$, i.e., 200 holds, then both $P_{1}$ and $P_{2}$ go extinct in (23);

- if $R_{0}>1$, i.e., (21) holds, there is uniform persistence of $P_{1}+P_{2}$ for (23).

In [29], a nonautonomous model of this type was analyzed, namely

$$
\left\{\begin{array}{l}
x_{1}^{\prime}=x_{1}\left(a_{1}-b_{1} x_{1}\right)-\frac{c_{2} x_{1} x_{2}}{\alpha+\beta x_{1}+\gamma x_{2}}-\frac{c_{3} x_{1} x_{3}}{\alpha+\beta x_{1}+\gamma x_{3}} \\
x_{2}^{\prime}=x_{2}\left(-a_{2}+\frac{d_{2} x_{1}}{\alpha+\beta x_{1}+\gamma x_{2}}-b_{2} x_{3}\right) \\
x_{3}^{\prime}=x_{3}\left(-a_{3}+\frac{d_{3} x_{1}}{\alpha+\beta x_{1}+\gamma x_{3}}-b_{3} x_{2}\right)
\end{array}\right.
$$

where $x_{1}$ is the prey and $x_{2}, x_{3}$ are the predators (observe that here the interaction between the predators is of competitive type). In that paper, some sufficient conditions guaranteeing uniform persistence for all the species were given, in terms of lower and upper bounds on the coefficients. In the last section therein, the existence of a periodic solution was proved assuming that the coefficients are periodic.

Wishing to compare such a result with Proposition 4.4 here we assume that the coefficients $a_{i}(\cdot), b_{i}(\cdot), c_{i}(\cdot), d_{i}(\cdot)$ (for $\left.i=1,2,3\right)$ and $\alpha(\cdot), \beta(\cdot), \gamma(\cdot)$ are positive, continuous and $T$-periodic functions. Our result gives simpler and weaker conditions, allowing on the other hand to obtain less information, namely only the uniform persistence of $P_{1}+P_{2}$. In the case of constant coefficients, for instance, we have $x_{1}^{*}=a_{1} / b_{1}$ so that, using the notation in [29, Theorem 3.2], we can take as lower and upper bound for $x_{1}^{*}$ the number $m_{1}^{0}=a_{1} / b_{1}=M_{1}^{0}$. Hence, our condition ensuring uniform persistence of $x_{2}+x_{3}$ reads as

$$
\max \left\{\frac{d_{2} m_{1}^{0}}{a_{2}\left(\alpha+\beta m_{1}^{0}\right)}, \frac{d_{3} m_{1}^{0}}{a_{3}\left(\alpha+\beta m_{1}^{0}\right)}\right\}>1,
$$

while [29, Hypothesis 3.1], yielding uniform persistence of both $x_{2}$ and $x_{3}$, is given by

$$
\frac{d_{2} m_{1}^{0}-\left(a_{2}+b_{2} M_{3}^{0}\right)\left(\beta m_{1}^{0}+\alpha\right)}{\left(a_{2}+b_{2} M_{3}^{0}\right) \gamma}>0, \quad \frac{d_{3} m_{1}^{0}-\left(a_{3}+b_{3} M_{2}^{0}\right)\left(\beta m_{1}^{0}+\alpha\right)}{\left(a_{3}+b_{3} M_{2}^{0}\right) \gamma}>0,
$$

i.e., the stronger assumption

$$
\frac{d_{2} m_{1}^{0}}{\left(a_{2}+b_{2} M_{3}^{0}\right)\left(\alpha+\beta m_{1}^{0}\right)}>1, \quad \frac{d_{3} m_{1}^{0}}{\left(a_{3}+b_{3} M_{2}^{0}\right)\left(\alpha+\beta m_{1}^{0}\right)}>1,
$$

where $M_{2}^{0}$ and $M_{3}^{0}$ are upper bounds for $x_{2}$ and $x_{3}$, respectively. 
2) In the spirit of the previous case, we are able to admit interactions of more general type between the predators. In particular, we separately comment the situation involving models of prey-mesopredator-superpredator kind, like in [10, 33. As an example, assume that a natural system is composed by a single prey population (birds) $B$ and a mesopredator one (rats) $R$, which preys $B$ and is preyed by a superpredator species $C$ (cats). This can be modeled by the system appearing in [10, formula (2.2)], of which we propose the following nonautonomous generalization:

$$
\left\{\begin{array}{l}
B^{\prime}=r(t) B\left(1-\frac{B}{K(t)}\right)-\frac{a_{1}(t) B R}{1+\delta(t) B+\sigma(t) R}-\frac{a_{2}(t) B C}{1+\alpha(t) B+\beta(t) R+\gamma(t) C} \\
R^{\prime}=\frac{b_{1}(t) B R+b_{2}(t) R}{1+\delta(t) B+\sigma(t) R}-d_{r}(t) R-\frac{b_{3}(t) R C}{1+\alpha(t) B+\beta(t) R+\gamma(t) C} \\
C^{\prime}=-d_{c}(t) C+\frac{c_{1}(t) B C+c_{2}(t) R C}{1+\alpha(t) B+\beta(t) R+\gamma(t) C},
\end{array}\right.
$$

where all the considered coefficients are positive and continuous and may depend on time in a $T$-periodic way. We recall that the appearance of the term $\frac{b_{2}(t) R}{1+\delta(t) B+\sigma(t) R}$ depends on the fact that rats can also consume non-avian food [10].

We first observe that if either $R=0$ or $C=0$, we find a predator-prey model of the kind treated in Section 3 having Beddington-De Angelis functional response. On the other hand, if $R=C=0$, the equation for $B$ has a $T$-periodic solution $B^{*}(t)$, as is well known [14, Example 4.19]. In our approach, this suggests to search for a uniform persistence result for the species $R$ and $C$. Correspondingly, we have

$$
F(t)=\left[\begin{array}{cc}
\frac{b_{1}(t) B^{*}+b_{2}(t)}{1+\delta(t) B^{*}} & 0 \\
0 & \frac{c_{1}(t) B^{*}}{1+\alpha(t) B^{*}}
\end{array}\right]
$$

and

$$
V(t)=\left[\begin{array}{cc}
d_{r}(t) & 0 \\
0 & d_{c}(t)
\end{array}\right]
$$

Also in this case the matrices $F(t)$ and $V(t)$ are diagonal, and this allows to write easily the condition concerning the basic reproduction number. We thus have the following proposition.

Proposition 4.5. Let $R_{0}$ be the basic reproduction number for model (24), defined as in Section 2, namely

$$
R_{0}=\max \left\{\frac{\int_{0}^{T} \frac{b_{1}(t) B^{*}+b_{2}(t)}{1+\delta(t) B^{*}} d t}{\int_{0}^{T} d_{r}(t) d t}, \frac{\int_{0}^{T} \frac{c_{1}(t) B^{*}}{1+\alpha(t) B^{*}} d t}{\int_{0}^{T} d_{c}(t) d t}\right\} .
$$

We have the following:

- if $R_{0}<1$, then $R$ and $C$ go extinct;

- if $R_{0}>1$, then there is uniform persistence of $R+C$.

If the considered coefficients are constant, the solution $B^{*}(t)$ corresponds to the equilibrium $K$, so that uniform persistence of $R+C$ is obtained as long as

$$
d_{r}<\frac{b_{1} K+b_{2}}{1+\delta K}, \quad \text { or } \quad d_{c}<\frac{c_{1} K}{1+\alpha K}
$$


Notice that it is not possible, in general, to obtain better results, as is shown also in 10 , Figure 3.2 (a)]. The growth of rats and cats can be controlled artificially, as well, introducing an external death factor $-\mu_{r} R$ and $-\mu_{c} C$ leading to the so called controlled version of the bird-rat-cat system [10, formula (4.1)], which displays similar dynamical features.

3) We can also consider a model with stage structure for the predators, namely a situation in which the predator is present in form of offsprings and adult individuals. Precisely, denoting by $P_{1}$ the amount of immature predator and by $P_{2}$ the amount of mature predator, we can consider the corresponding model

$$
\left\{\begin{array}{l}
N^{\prime}=k(t, N) N-g\left(t, N, P_{2}\right) P_{2} \\
P_{1}^{\prime}=-\xi_{1}(t) P_{1}-\eta_{1}(t) P_{1}^{2}+\beta(t) g\left(t, N, P_{2}\right) P_{2}-\delta(t) P_{1} \\
P_{2}^{\prime}=-\xi_{2}(t) P_{2}-\eta_{2}(t) P_{2}^{2}+\delta(t) P_{1},
\end{array}\right.
$$

where $k: \mathbb{R} \times \mathbb{R}_{+} \rightarrow \mathbb{R}$ is continuous and $T$-periodic in the $t$-variable and differentiable in $N$ and $g: \mathbb{R} \times \mathbb{R}_{+}^{2} \rightarrow \mathbb{R}_{+}, \beta, \delta, \xi_{i}, \eta_{i}: \mathbb{R} \rightarrow \mathbb{R}_{+}, i=1,2$, are continuous functions, $T$-periodic in the $t$-variable and differentiable in the other variables (if depending explicitly on them). We also assume that $\delta(t)$ is strictly positive, meaning that offsprings become adult at a certain positive rate. In this case, we have

$$
F(t)=\left[\begin{array}{cc}
0 & \beta(t) g\left(t, N^{*}(t), 0\right) \\
0 & 0
\end{array}\right],
$$

and

$$
V(t)=\left[\begin{array}{cc}
\xi_{1}(t)+\delta(t) & 0 \\
-\delta(t) & \xi_{2}(t)
\end{array}\right],
$$

and we cannot obtain explicitly $R_{0}$, see [2, 32]. Nevertheless, we have the following.

Proposition 4.6. Let $R_{0}$ be the basic reproduction number for (25), defined as in Section 2 . Then,

- if $R_{0}<1$, then both $P_{1}$ and $P_{2}$ go extinct;

- if $R_{0}>1$, there is uniform persistence of both $P_{1}$ and $P_{2}$.

We omit the proof as it is similar to the previous ones. We only mention that once uniform persistence of $P_{1}+P_{2}$ is obtained under the assumption that $R_{0}>1$, we have that there exists $\sigma>0$ such that $P_{1}(t) \geq \frac{\sigma}{2}-P_{2}(t)$ for every $t \geq t_{0}$, for a suitable $t_{0}>0$. Inserting this inequality into the equation for $P_{2}$ yields

$$
P_{2}^{\prime} \geq \delta(t) \frac{\sigma}{2}-\left(\delta(t)+\xi_{2}(t)\right) P_{2}-\eta_{2}(t) P_{2}^{2},
$$

from which it follows that $P_{2}$ is bounded from below by a positive constant. Indeed, observing that $P_{2}^{2} \leq P_{2}$ if $P_{2} \leq 1$, it follows that

$$
P_{2}(t) \geq \min \left\{1, \frac{\sigma \min _{t \in[0, T]} \delta(t)}{4 \max _{t \in[0, T]}\left(\delta(t)+\xi_{2}(t)+\eta_{2}(t)\right)}\right\}>0 .
$$

The persistence for $P_{1}$ now follows as in the proof of Theorem 3.2 
This proposition applies, for instance, to the system

$$
\left\{\begin{array}{l}
x^{\prime}=x(r(t)-a(t) x)-y_{2}\left(\frac{b(t) x}{x+c(t)}\right) \\
y_{1}^{\prime}=-\left(D(t)+d_{1}(t)\right) y_{1}+k y_{2}\left(\frac{b(t) x}{x+c(t)}\right) \\
y_{2}^{\prime}=D(t) y_{1}-d_{2}(t) y_{2}
\end{array}\right.
$$

an autonomous version of which was analyzed in [13, Example 1], where the authors studied the domain of stability of the nontrivial equilibrium. In that paper, it is shown that when $R_{0}>1$ and the limit at infinity of the preys is sufficiently large, the positive equilibrium is globally stable. Moreover, it is there given a uniform persistence result for the autonomous case which we are able to recover and extend to the nonautonomous setting.

Remark 4.7. Similarly as in Remark 3.5. we can deal all the same with models with more preys and more predators, admitting passages between the species, provided that the predator-free system behaves according to Proposition 3.6. For instance, we can admit models with more preys and more predators with stage structure for both species. Even more, we can analyze, for instance, a system like

$$
\left\{\begin{array}{l}
N_{1}^{\prime}=a(t) N_{2}-b(t) N_{1}-d(t) N_{1}^{2}(t)-f_{1}\left(t, N_{1}, P_{1}\right) P_{1}-g_{1}\left(t, N_{1}, P_{2}\right) P_{2} \\
N_{2}^{\prime}=c(t) N_{1}-f(t) N_{2}^{2}(t)-f_{2}\left(t, N_{2}, P_{1}\right) P_{1}-g_{2}\left(t, N_{2}, P_{2}\right) P_{2} \\
P_{1}^{\prime}=-\xi_{1}(t) P_{1}-\eta_{1}(t) P_{1}^{2}-\delta_{1}(t) P_{1}+\alpha_{1}(t) f_{1}\left(t, N_{1}, P_{1}\right) P_{1}+\alpha_{2}(t) f_{2}\left(t, N_{2}, P_{1}\right) P_{1} \\
P_{2}^{\prime}=-\xi_{2}(t) P_{2}-\eta_{2}(t) P_{2}^{2}+\delta_{1}(t) P_{1}+\beta_{1}(t) g_{1}\left(t, N_{1}, P_{2}\right) P_{2}+\beta_{2}(t) g_{2}\left(t, N_{2}, P_{2}\right) P_{2} .
\end{array}\right.
$$

First of all, notice that by [7, Theorem 4] the system

$$
\left\{\begin{array}{l}
N_{1}^{\prime}=a(t) N_{2}-b(t) N_{1}-d(t) N_{1}^{2}(t) \\
N_{2}^{\prime}=c(t) N_{1}-f(t) N_{2}^{2}(t)
\end{array}\right.
$$

admits a unique globally asymptotically stable $T$-periodic orbit $\left(N_{1}^{*}(t), N_{2}^{*}(t)\right)$. Moreover, we have

$$
F(t)=\left[\begin{array}{cc}
\alpha_{1}(t) f_{1}\left(t, N_{1}^{*}(t), 0\right)+\alpha_{2}(t) f_{2}\left(t, N_{2}^{*}(t), 0\right) & 0 \\
0 & \beta_{1}(t) g_{1}\left(t, N_{1}^{*}(t), 0\right)+\beta_{2}(t) g_{2}\left(t, N_{2}^{*}(t), 0\right)
\end{array}\right],
$$

and

$$
V(t)=\left[\begin{array}{cc}
\xi_{1}(t)+\delta_{1}(t) & 0 \\
-\delta_{1}(t) & \xi_{2}(t)
\end{array}\right]
$$

and we can obtain similar results as in the previous example 3$)$.

In this perspective, in [16, 17. models with two preys $x_{1}, x_{2}$ and two predators $y_{1}, y_{2}$, such that $y_{1}$ attacks $x_{1}$ and $y_{2}$ attacks $x_{2}$ (namely setting $f_{2}(t, N, P) \equiv g_{1}(t, N, P) \equiv 0$ ), with no interaction between the predators, were analyzed. It seems that there are some problems with the proofs in those papers, since the reasonings leading therein to persistence do not cover all the possible scenarios, so that it does not seem possible, in general, to deduce uniform persistence of both predators. With our results, on the other hand, the uniform persistence of $y_{1}+y_{2}$ is guaranteed and, possibly, different functional responses can be considered for $x_{1}$ and $x_{2}$.

\section{Models of Leslie-Gower type}

So far, we have considered models for which the response to the impact of predation is symmetric, coupling the functional and the numerical response of predators to prey density through a 
multiplicative factor. In this last section, we briefly analyze differential systems lacking this feature, as the ones considered in [1, 5. We choose to deal with this situation in a different section since here, as we will see, the roles of preys and predators are interchanged; this is a further reason justifying the general setting under which we formulated the abstract results of Section 2, so as to make them as applicable and flexible as possible.

More precisely, we consider a Leslie-Gower model (possibly with mutual interference as in [31]) described by a system like

$$
\left\{\begin{array}{l}
x^{\prime}=c_{1}(t) x\left(1-\frac{x}{H(t)}\right)-f(x, y) y \\
y^{\prime}=c_{2}(t) y\left(1-\frac{y}{x+c(t)}\right)
\end{array}\right.
$$

where $x$ is the prey and $y$ the predator, and $f(0, y) \equiv 0$. For simplicity, we assume that $c_{i}(\cdot)$, $i=1,2, H(\cdot), c(\cdot)$, are positive $T$-periodic functions (but we could assume that $c_{i}(\cdot)$ vanishes in some intervals, $i=1,2)$.

As a first observation, notice that, if the functions $c(t), c_{1}(t), c_{2}(t), H(t)$ are constant and $x \equiv 0$, the predator does not die, but it goes asymptotically towards the equilibrium $y \equiv c$. Indeed, these models become biologically meaningful if we admit that the predator switches over to other populations in case of scarcity of the prey. However, as it is intuitive, the larger the prey, the larger the growth of the predator.

From the equation, we immediately see that if $y(0)>0$, then $\liminf _{t \rightarrow+\infty} y(t) \geq \min _{t \in[0, T]} c(t)$, so that persistence of the predator appears as an easy matter. Our aim is thus to prove persistence of the preys, exploiting the strategy of the previous sections (using in particular Theorem 3.2).

To this end, we first notice that the prey-free system admits a periodic solution $\left(0, y^{*}\right)$; moreover, we have that

$$
F(t)=c_{1}(t), \quad V(t)=\frac{\partial f}{\partial x}\left(0, y^{*}\right) y^{*} .
$$

Assumptions $\left(A_{1}\right)-\left(A_{5}\right)$ are then easily shown to hold, while, in order for $\left(A_{6}\right)$ to be fulfilled, we have to require that

$$
\int_{0}^{T} \frac{\partial f}{\partial x}\left(0, y^{*}\right) y^{*} d t>0
$$

On the other hand, $\left(A_{7}\right)$ is satisfied for

$$
\mathcal{K}=\left\{(x, y) \in \mathbb{R}_{+}^{2} \mid x \leq \max _{t \in[0, T]} H(t)+1, y \leq \max _{t \in[0, T]} H(t)+\max _{t \in[0, T]} c(t)+1\right\} .
$$

Finally, $R_{0}>1$ corresponds to

$$
\int_{0}^{T} c_{1}(t) d t>\int_{0}^{T} \frac{\partial f}{\partial x}\left(0, y^{*}\right) y^{*} d t
$$

Thus, assuming (26) and 27) leads us to obtain uniform persistence of $x(t)$ as in the previous sections.

We now conclude the paper by restating this last result with more details in the framework of the model studied in [1], namely

$$
\left\{\begin{array}{l}
x^{\prime}=x\left(1-x-\frac{y}{(1+A x)(1+B y)}\right) \\
y^{\prime}=C y\left(1-\frac{D y}{x+E}\right)
\end{array}\right.
$$


of which we are going to consider a periodic version. Observe preliminarily that if $A, B, C, D, E$ are constant, then the equation for $y$ gives the positive asymptotically stable prey-free solution $y(t) \equiv E / D$. Since in this case $F(t)=1$ and

$$
V(t)=\frac{E}{D+B E},
$$

uniform persistence is obtained when

$$
D+B E>E,
$$

as in 1 .

Let us now assume that $A, B, C, D, E: \mathbb{R} \rightarrow \mathbb{R}$ are positive, continuous and $T$-periodic functions. We first observe that the existence of a globally asymptotically stable positive periodic solution $y^{*}(t)$ to

$$
y^{\prime}=C(t) y\left(1-\frac{D(t) y}{E(t)}\right)
$$

is a consequence of [14, Example 4.19 and Exercise 4.21]. We now have the following result.

Proposition 5.1. If

$$
\int_{0}^{T} 1-\frac{y^{*}(t)}{1+B(t) y^{*}(t)} d t>0
$$

we have uniform persistence of both $x(t)$ and $y(t)$ for model 28 .

Indeed,

- 260 is trivially satisfied;

- condition 29 corresponds to $R_{0}>1$ for our model.

We can thus use Theorem 3.2 to conclude uniform persistence of the prey.

\section{Appendix}

In this Appendix, we recall (without proofs) some of the abstract results from [19] which have been used throughout the proofs of Lemma 3.1 and Proposition 3.6 above, adapting their statements to our particular situation. We choose to state them explicitly in order to ease the readability of the paper, since, differently from the other abstract results we refer to, maybe it appears less easy to have access to them.

Our object of interest is a system of the type

$$
\left\{\begin{array}{l}
u_{1}^{\prime}=U_{1}(t, u) \\
\cdots \\
u_{n}^{\prime}=U_{n}(t, u),
\end{array} \quad u=\left(u_{1}, \ldots, u_{n}\right),\right.
$$

where the functions $U_{i}: \mathbb{R} \times \mathbb{R}^{n} \rightarrow \mathbb{R}, i=1, \ldots, n$, are continuous, continuously differentiable in the $u$-variable, and $\omega$-periodic in the $t$-variable, $\omega>0$.

In our setting, only nonnegative solutions are meaningful, so that we restrict our attention to initial conditions belonging to the cone

$$
\mathbb{R}_{+}^{n}=\left\{u \in \mathbb{R}^{n}: u_{i} \geq 0, i=1, \ldots, n\right\} .
$$


We assume that the solutions to the Cauchy problems starting in such cone are all global (namely, they are defined for every $t \in[0, \omega]$ ); notice that this is the case in Lemma 3.1 and Proposition 3.6. in view of the presence of a compact global attractor. Moreover, since local uniqueness holds thanks to the local Lipschitz continuity with respect to the $u$-variable, we can associate with (30) a Poincaré operator, defined by

$$
\mathcal{U}(\omega)\left(u_{0}\right)=u\left(\omega ; 0, u_{0}\right)
$$

where $u\left(t ; 0, u_{0}\right)$ is the unique solution to the Cauchy problem associated with 30 having initial datum $u\left(0 ; 0, u_{0}\right)=u_{0}$. Obviously, $\mathcal{U}(\omega): \mathbb{R}_{+}^{n} \rightarrow \mathbb{R}^{n}$. The first lemma we recall gives a condition for which $\mathcal{U}(\omega): \mathbb{R}_{+}^{n} \rightarrow \mathbb{R}_{+}^{n}$, exploiting the following property of positiveness.

Definition 6.1. ([19, \$4.4]) We say that the right-hand side in (30) has the property of positiveness if, for every $i=1, \ldots, n$, it holds

$$
U_{i}\left(t, \hat{u}_{i}\right) \geq 0,
$$

where

$$
\hat{u}_{i}=\left(u_{1}, \ldots, u_{i-1}, 0, u_{i+1}, \ldots, u_{n}\right),
$$

with $u_{j} \geq 0$ for every $j \in\{1, \ldots, n\} \backslash\{i\}$. We say that the right-hand side in (30) has the property of strong positiveness if the inequality in (31) is strict (provided that at least one component $u_{j}$ is positive in $(32), j \neq i)$.

Lemma 6.2. ([19, Lemma 4.1]) If the right-hand side in (30) has the property of positiveness, then every initial condition $u_{0} \in \mathbb{R}_{+}^{n}$ gives rise to a nonnegative solution $u(t)$ to $(30)$.

It follows that, for every $\omega^{\prime} \in[0, \omega]$, it holds $\mathcal{U}\left(\omega^{\prime}\right): \mathbb{R}_{+}^{n} \rightarrow \mathbb{R}_{+}^{n}$. In particular, the operator $\mathcal{U}(\omega)$ is positive.

In the following definition, we now list some other properties of general operators which will be useful in order to recall the other abstract results from [19] which have been used throughout the paper.

Definition 6.3. ([19, p. 193]) Let $\mathfrak{U}$ be a nonlinear operator defined on the cone $\mathbb{R}_{+}^{n}$. We say that $\mathfrak{U}$ is

- monotonic, if $0 \leq a \leq b$ implies $\mathfrak{U}(a) \leq \mathfrak{U}(b)$ (here the ordering is the one induced by the cone $\left.\mathbb{R}_{+}^{n}\right)$;

- strongly positive, if for $0 \neq a \in \mathbb{R}_{+}^{n}$ it is $\mathfrak{U}(a) \in \operatorname{int} \mathbb{R}_{+}^{n}$;

- strongly concave on $\mathbb{R}_{+}^{n}$, if for every $a \in \operatorname{int} \mathbb{R}_{+}^{n}$ and every $c_{1} \in(0,1)$ there exists $c_{2}>0$ with

$$
\mathfrak{U}\left(c_{1} a\right) \geq\left(1+c_{2}\right) c_{1} \mathfrak{U}(a) .
$$

The validity of such abstract conditions is related with the properties of the right-hand side in system (30) by [19, Theorem 10.2], which we here recall.

Proposition 6.4. ([19, Theorem 10.2]) If the right-hand side in (30) has the property of positiveness, and, for every $i=1, \ldots, n$, the functions

$$
h_{i}(t, u):=U_{i}(t, u)-\sum_{j=1}^{n} u_{j} \frac{\partial U_{i}(t, u)}{\partial u_{j}}
$$

are nonnegative for $(t, u) \in[0, \omega] \times \operatorname{int} \mathbb{R}_{+}^{n}$ and strictly positive for $t=0$ and $u \in \operatorname{int} \mathbb{R}_{+}^{n}$, then the translation operator $\mathcal{U}(\omega)$ associated with (30) is monotonic, strongly positive and strongly concave on $\mathbb{R}_{+}^{n}$. 
If an operator fulfills all the conditions in Definition 6.3 (in particular, this is the case for a system satisfying the assumptions of Proposition 6.4), it is possible to prove uniqueness of its fixed points (if any), as highlighted by the following proposition. Of course, since fixed points of $\mathcal{U}(\omega)$ correspond to $\omega$-periodic solutions to $(30)$, this statement has a certain importance in understanding the dynamics of the considered system.

Proposition 6.5. ([19, Theorem 10.1]) A monotonic strongly positive and strongly concave operator $\mathfrak{U}$ defined on $\mathbb{R}_{+}^{n}$ has at most one fixed point in $\mathbb{R}_{+}^{n}$.

As for the existence of a fixed point, in general it is established by means of dynamical, topological or functional techniques: for instance, in Lemma 3.1 we have used the well known properties of $\omega$ periodic 1-dimensional differential equations [14, Theorem 4.11], as well as Massera's Theorem for 2-dimensional ones in Proposition 3.6. Once an $\omega$-periodic solution to (30) exists, it is then natural, as a further step, to examine its stability. We conclude this appendix by recalling, in this respect, the following statement.

Proposition 6.6. ([19, Theorem 10.6 and remark $p$. 213]) Assume that system (30) has a nonzero nonnegative $\omega$-periodic solution $u^{*}(t)$ with range in $\mathbb{R}_{+}^{n}$ and suppose that the translation operator $\mathcal{U}(\omega)$ associated with system (30) is monotonic, strongly positive and strongly concave on $\mathbb{R}_{+}^{n}$ (so that $u^{*}(0)$ is its unique fixed point by the previous proposition). Then, $u^{*}(t)$ is uniformly asymptotically stable. Moreover, it is also globally stable, in the sense that all the solutions $u\left(t ; 0, u_{0}\right)$ to 30 starting with a nonzero initial condition $u_{0} \in \mathbb{R}_{+}^{n}$ satisfy $\lim _{t \rightarrow+\infty}\left\|u\left(t ; 0, u_{0}\right)-u^{*}(t)\right\|=0$.

Acknowledgement. The authors wish to thank the anonymous referees for their careful reading of the paper and their suggestions which have helped improve its readability. They also want to thank Nicolas Bacaër for some useful discussions about this subject.

\section{References}

[1] Ali N, Jazar M (2013) Global dynamics of a modified Leslie-Gower predator-prey model with Crowley-Martin functional responses. J Appl Math Comput 43:271-293

[2] Bacaër N, Guernaoui S (2006) The epidemic threshold of vector-borne diseases with seasonality. J Math Biol 53:421-436

[3] Bacaër N, Ait Dads EH (2011) Genealogy with seasonality, the basic reproduction number, and the influenza pandemic. J Math Biol 62:741-762

[4] Bacaër N (2007) Approximation of the basic reproduction number $R_{0}$ for vector-borne diseases with a periodic vector population. Bull Math Biol 69:1067-1091

[5] Chen F, Chen L, Xie X (2009) On a Leslie-Gower predator-prey model incorporating a prey refuge. Nonlinear Anal Real World Appl 10:2905-2908

[6] Cui J, Chen L (1998) The Effect of Diffusion on the Time Varying Logistic Population Growth. Computers Math Applic 36:1-9

[7] Cui J, Chen L, Wang W (2000) The Effect of Dispersal on Population Growth with Stage-Structure. Computers Math Applic 39:91-102

[8] Cui J, Takeuchi Y (2006) Permanence, extinction and periodic solution of predator-prey system with BeddingtonDe Angelis functional response. J Math Anal Appl 317:464-474

[9] Cushing JM (1977) Periodic time-dependent predator-prey systems. SIAM J Appl Math 32:82-95

[10] Fan M, Kuang Y, Feng Z (2005) Cats protecting birds revisited. Bull Math Biol 67:1081-1106

[11] Fonda A (1988) Uniformly persistent semidynamical systems. Proc Amer Math Soc 104:111-116

[12] Georgescu P, Hsieh Y (2007) Global dynamics of a predator-prey model with stage structure for the predator. SIAM J Appl Math 67:1379-1395

[13] Georgescu P, Hsieh Y, Zhang H (2010) A Lyapunov functional for a stage-structured predator-prey model with nonlinear predation rate. Nonlinear Anal Real World Appl 11:3653-3665

[14] Hale J, Kocak H (1996) Dynamics and bifurcations. Springer New York

[15] Huang M, Li X (2011) Dispersal permanence of a periodic predator-prey system with Holling type-IV functional response. Appl Math Comput 218:502-513

[16] Huang C, Zhao M, Huo H (2008) Permanence of periodic predator-prey system with functional responses and stage structure for the prey. Abstr Appl Anal 2008, ID 371632:1-15 
[17] Huang C, Zhao M, Zhao L (2010) Permanence of periodic predator-prey system with two predators and stage structure for prey. Nonlinear Anal Real World Appl 11:503-514

[18] Kazarinov N, van den Driessche P (1978) A model predator-prey system with functional response. Math Biosci 39:125-134

[19] Krasnosel'skii MA (1968) The operator of translation along the trajectories of differential equations. Translations of Mathematical Monographs. Volume 19. AMS Providence Rhode Island

[20] Kuznetsov Y, Muratori S, Rinaldi S (1992) Bifurcations and chaos in a periodic predator-prey model. Int J Bifurcation Chaos 2:117-128

[21] Leray J, Schauder J (1934) Topologie et équations fonctionnelles. Annales Scient Éc Norm Sup 51:45-78

[22] Nelson WA, Bjørnstad ON, Yamanaka T (2013) Recurrent insect outbreaks caused by temperature-driven changes in system stability. Science 341:796-799

[23] Rebelo C, Margheri A, Bacaër N (2012) Persistence in seasonally forced epidemiological models. J Math Biol 64:933-949

[24] Rebelo C, Margheri A, Bacaër N (2014) Persistence in some periodic epidemic models with infection age or constant periods of infection. Discrete Cont Dyn Syst B 19:1155-1170

[25] Rinaldi S, Muratori S, Kuznetsov Y (1993) Multiple attractors, catastrophes and chaos in seasonally perturbed predator-prey communities. Bull Math Biol 55:15-35

[26] Ruiz-Herrera A (2011) Permanence of two species and fixed point index. Nonlinear Anal 74:146-153

[27] Smith HL, Thieme HR (2011) Dynamical systems and population persistence. American Mathematical Society, Providence RI

[28] Taylor RA, White A, Sherratt JA (2013) How do variations in seasonality affect population cycles? Proc Roy Soc B 280, ID 20122714:1-6

[29] Ton TV, Hieu NT (2011) Dynamics of species in a model with two predators and one prey. Nonlinear Anal $74: 4868-4881$

[30] van den Driessche P, Watmough J (2002) Reproduction numbers and sub-threshold endemic equilibria for compartmental models of disease transmission. Math Biosci 180:29-48

[31] Wang K (2011) Permanence and global asymptotical stability of a predator-prey model with mutual interference. Nonlinear Anal Real World Appl 12:1062-1071

[32] Wang W, Zhao X (2008) Threshold dynamics for compartmental epidemic models in periodic environments. J Dyn Differ Equ 20:699-717

[33] Zhang J, Fan M, Kuang Y (2006) Rabbits killing birds revisited. Math Biosci 203:100-123

[34] Zhang F, Zhao XQ (2007) A periodic epidemic model in a patchy environment. J Math Anal Appl 325:496-516

[35] Zhao XQ (2003) Dynamical systems in population biology. CMS Books in Mathematics 